\title{
Gravitational wave signatures of the absence of an event horizon: Nonradial oscillations of a thin-shell gravastar
}

\author{
Paolo Pani, ${ }^{1, *}$ Emanuele Berti, ${ }^{2,3, \dagger}$ Vitor Cardoso, ${ }^{2,4,+}$ Yanbei Chen, ${ }^{3,8}$ and Richard Norte ${ }^{3, \|}$ \\ ${ }^{1}$ Dipartimento di Fisica, Università di Cagliari, and INFN sezione di Cagliari, Cittadella Universitaria 09042 Monserrato, Italy \\ ${ }^{2}$ Department of Physics and Astronomy, The University of Mississippi, University, Mississippi 38677-1848, USA \\ ${ }^{3}$ Theoretical Astrophysics 350-17, California Institute of Technology, Pasadena, California 91125, USA \\ ${ }^{4}$ Centro Multidisciplinar de Astrofísica - CENTRA, Departamento de Física, Instituto Superior Técnico, Av. Rovisco Pais 1, \\ 1049-001 Lisboa, Portugal
}

(Received 1 September 2009; published 30 December 2009)

\begin{abstract}
Gravitational waves from compact objects provide information about their structure, probing deep into strong-gravity regions. Here we illustrate how the presence or absence of an event horizon can produce qualitative differences in the gravitational waves emitted by ultracompact objects. In order to set up a straw-man ultracompact object with no event horizon, but which is otherwise almost identical to a black hole, we consider a nonrotating thin-shell model inspired by Mazur and Mottola's gravastar, which has a Schwarzschild exterior, a de Sitter interior and an infinitely thin shell with finite tension separating the two regions. As viewed from the external space-time, the shell can be located arbitrarily close to the Schwarzschild radius, so a gravastar might seem indistinguishable from a black hole when tests are only performed on its external metric. We study the linearized dynamics of the system, and, in particular, the junction conditions connecting internal and external gravitational perturbations. As a first application of the formalism we compute polar and axial oscillation modes of a thin-shell gravastar. We show that the quasinormal mode spectrum is completely different from that of a black hole, even in the limit when the surface redshift becomes infinite. Polar quasinormal modes depend on the equation of state of matter on the shell and can be used to distinguish between different gravastar models. Our calculations suggest that low-compactness gravastars could be unstable when the sound speed on the shell $v_{s} / c \gtrsim 0.92$.
\end{abstract}

DOI: 10.1103/PhysRevD.80.124047

PACS numbers: 04.30.Db, 04.25.Nx, 04.80.Cc

\section{INTRODUCTION}

Black holes (BHs), once considered an exotic mathematical solution to Einstein's field equations, have now been widely accepted as astronomical objects [1-4]. Stellar-mass BHs are believed to be the final stage of the evolution of sufficiently massive stars. Massive BHs seem to populate the center of many galaxies at low redshift, and must have played an important role in the formation of structure in the Universe. Most evidence supporting the astrophysical reality of BHs comes from the weak-gravity region, i.e. from observations probing the space-time several Schwarzschild radii away from the event horizon. Attempts to rule out possible alternatives to BHs usually rely on general relativity being the correct theory of gravity, and/or on constraints on the equation of state of matter at high densities. For massive BHs the most precise measurements so far come from observations of stellar proper motion at the center of our own Galaxy, indicating the

\footnotetext{
*paolo.pani@ca.infn.it

Currently at Centro Multidisciplinar de Astrofísica - CENTRA, Departamento de Física, Instituto Superior Técnico, Av. Rovisco Pais 1, 1049-001 Lisboa, Portugal

†berti@phy.olemiss.edu

\#vitor.cardoso@ist.utl.pt

$\S$ yanbei@tapir.caltech.edu

"norte@ caltech.edu
}

presence of a "dark object" of mass $M \simeq(4.1 \pm 0.6) \times$ $10^{6} M_{\odot}$ [5]. Recent millimeter and infrared observations of Sagittarius $A^{*}$, the compact source of radio, infrared, and $\mathrm{X}$-ray emission at the center of the Milky Way, infer an intrinsic diameter of $37_{-10}^{+16}$ microarcseconds, even smaller than the expected apparent size of the event horizon of the presumed BH [6]. Some of the exotic alternatives to a BH (such as "fermion balls") are incompatible with the observations [7] and any distribution of individual objects within such a small region (with the possible exception of dark matter particles or asteroids, which however should be ejected by three-body interactions with stars) would be gravitationally unstable $[8,9]$. In a recent attempt to probe the event horizon, Broderick and Narayan have analyzed the observations of Ref. [6]. If the object at the center of our Galaxy had a surface it would be hot enough to glow with a steady emission of infrared light, but no such glow has been detected [10]. This and similar arguments are inevitably dependent on the gas distribution and on details of the accretion process, and they really set lower limits on the gravitational redshift corresponding to the hypothetical surface replacing the event horizon (see e.g. [11] for a review). Indeed, some hold the view that an observational proof of the existence of event horizons based on electromagnetic observations is fundamentally impossible [12]. For these reasons, model-independent tests of the strongfield dynamics of $\mathrm{BHs}$ and studies of the gravitational 
radiation signatures of event horizons are necessary to confirm or disprove the $\mathrm{BH}$ paradigm $[13,14]$.

Gravitational wave detectors offer a new way of observing BHs, complementing the wealth of information from present electromagnetic observations [4]. As first proposed by Ryan [15,16], an exquisite map of the external spacetime of BHs (outside the innermost stable orbit, if there is any) can be constructed by observing the gravitational waveform emitted when a small compact object spirals into the putative supermassive $\mathrm{BH}$ at the center of a galaxy with the Laser Interferometer Space Antenna (LISA). As an extension of Ryan's work, Li and Lovelace considered the small object's tidal coupling with the central object and showed that by studying details of radiation reaction, information about the space-time region within the orbit can also be obtained [17]. Ryan's proposal to map space-times using inspiral waveforms is promising, but the data analysis task is affected by a "confusion problem": the possibility of misinterpreting truly non-Kerr waveforms by Kerr waveforms with different orbital parameters $[18,19]$. This ambiguity was shown to be resolvable if the orbit is known to be circular [15] or if one only probes the mass, spin, and quadrupole moment of the object using waveforms generated in the weak-gravity region [20]. A different approach to test the $\mathrm{BH}$ nature of ultracompact objects is based on measuring several of their free oscillation frequencies ("ringdown waves") and comparing them with the quasinormal mode (QNM) spectrum of BHs [21]. These tests of the "no-hair theorem" require a signal-to-noise ratio which could be achieved by advanced Earth-based gravitational wave interferometers and they are one of the most promising science goals of LISA [22-24].

In this paper we explore how to test possible alternatives to the $\mathrm{BH}$ paradigm. We retain the "conservative" assumption that general relativity is the correct theory of gravity and we focus on possible tests of the existence (or absence) of an event horizon.

Theorists conceived several families of compact objects with no event horizons. For example, boson stars are horizonless compact objects based on plausible models of particle physics at high densities, and they are (still) compatible with astrophysical observations [25,26]. Being indistinguishable from BHs in the Newtonian regime, boson stars are good "straw men" for supermassive BHs. The space-time of nonrotating spherical boson stars can approximate arbitrarily well a Schwarzschild geometry even close to the event horizon, and being very compact it is not easily distinguishable from a $\mathrm{BH}$ by electromagnetic observations [26,27]. Building on Ryan's proposal, Kesden et al. showed that the inspiral of a small compact object into a nonrotating boson star will emit a rather different gravitational waveform at the end of the evolution, when the small object falls into the central potential well of the boson star instead of disappearing into the event horizon of a BH [28]. Several authors have computed the
QNM spectrum of boson stars, showing that it is remarkably different from the QNM spectrum of BHs and lending support to the feasibility of no-hair tests using QNM measurements [29-31].

Another proposed alternative to massive $\mathrm{BHs}$, which we shall focus on in this paper, are the so-called gravastars [32]. The gravastar model assumes that the space-time undergoes a quantum vacuum phase transition in the vicinity of the $\mathrm{BH}$ horizon. The model effectively replaces the BH event horizon by a transition layer (or shell) and the BH interior by a segment of de Sitter space [33,34]. Mazur and Mottola argued for the thermodynamic stability of the model. A dynamical stability analysis by Visser and Wiltshire [35] confirmed that a simplified version of the gravastar model by Mazur and Mottola is also stable under radial perturbations for some physically reasonable equations of state for the transition layer. Chirenti and Rezzolla [36] first considered nonradial perturbations of gravastars, restricting attention to the relatively simple case of oscillations with axial parity. They computed the dominant axial oscillation modes and found no instabilities. In analogy with previous studies of the oscillation modes of boson stars [29-31], they confirmed that the axial QNM spectrum of gravastars can be used to discern a gravastar from a $\mathrm{BH}$. In the thin-shell limit, the axial QNM frequencies of Ref. [36] and our own calculations recover Fiziev's calculation of the axial QNMs of ultracompact objects with a totally reflecting surface [37].

In this paper we study the stability with respect to nonradial oscillations of a simplified "thin-shell" gravastar, retaining most of the essential features of the original model. We consider both axial perturbations (reproducing and extending the results of Ref. [36]) and polar perturbations. In the polar case, the matching of interior and exterior perturbations at the gravastar shell requires a more careful analysis because (unlike the axial case) polar perturbations of spherical objects actually induce motions of matter, which in turn couple back to gravitational perturbations. For this reason, nonspherical polar perturbations provide a more stringent test on the gravastar's overall stability. Polar perturbations are also crucial in studying the dynamics of objects orbiting the hypothetical gravastar. In order to treat polar perturbations of a nonrotating thin-shell gravastar we set up a rather generic formalism combining standard perturbation theory (in the Regge-Wheeler gauge) with Israel's junction conditions [38]. The formalism can be applied to gravitational perturbations of any spherically symmetric space-time characterized by regions with different cosmological constants separated by infinitely thin shells with finite surface energy and tension. Because matter is concentrated on these shells, the junction conditions deduced here will be sufficient in describing the linear dynamics of matter. Quite predictably, these conditions depend on the equation of state of the shell. As an application of the formalism we 
study the QNM spectrum of polar and axial perturbations of gravastars, exploring the nonradial stability of these objects. Polar QNMs (unlike axial QNMs) depend on the equation of state of matter on the shell: they can be used not only to distinguish a gravastar from a $\mathrm{BH}$, but also to distinguish between different gravastar models. We also find that the imaginary part of some QNMs seems to have a zero crossing when the gravastar is not very compact and the speed of sound on the shell is close to the speed of light, suggesting that some gravastar models may be unstable under nonradial perturbations. In a companion paper we will apply the formalism developed in this paper to study gravitational radiation from compact objects inspiraling into nonrotating gravastars.

The plan of the paper is as follows. In Sec. II we review our static thin-shell gravastar model. Section III sketches the calculation of axial and polar gravitational perturbations and of the matching conditions at the gravastar shell. Details of the matching procedure are provided in Appendix A, and details of the QNM calculation are given in Appendix B. Our numerical results for the polar and axial QNM spectra are presented in Sec. IV and supported by analytical calculations in the high-compactness limit in Appendix C.

We use geometrical units $(G=c=1)$. The Fourier transform of the perturbation variables is performed by assuming a time dependence of the form $e^{i \omega t}$. Greek indices $(\mu, \nu, \ldots)$ refer to the four-dimensional space-time metric. Latin indices $(i, j, \ldots)$ refer to the threedimensional space-time metric on the shell. Latin indices at the beginning of the alphabet $(a, b, \ldots)$ refer to the spatial metric on a two-sphere.

\section{EQUILIBRIUM MODEL}

The metric for a static thin-shell gravastar has the form $[35,36]$

$$
d s_{0}^{2}=-f(r) d t^{2}+\frac{1}{h(r)} d r^{2}+r^{2}\left(d \theta^{2}+\sin ^{2} \theta d \varphi^{2}\right) .
$$

Here

$$
f(r)= \begin{cases}h(r)=1-\frac{2 M}{r}, & r>a, \\ \alpha h(r)=\alpha\left(1-\frac{8 \pi \rho}{3} r^{2}\right), & r<a,\end{cases}
$$

where $M$ is the gravastar mass measured by an outside observer, and $\rho=3 M /\left(4 \pi a^{3}\right)$ is the "energy density" of the interior region. The coordinate system $(t, r, \theta, \varphi)$ has been chosen in such a way that the thin shell occupies a coordinate sphere with $r=a$. The space-time reduces to de Sitter for $r<a$, and to Schwarzschild for $r>a$. The junction conditions on the $r=a$ surface have already partially been chosen by requiring the induced metric to be continuous across the shell, which also dictates that $f(r)$ be continuous at $r=a$, or

$$
1-\frac{2 M}{a}=\alpha\left(1-\frac{8 \pi \rho a^{2}}{3}\right) \text {. }
$$

In this paper we shall usually drop the dependence of $f(r)$ and $h(r)$ on $r$. From the jump in the radial derivatives of $f$ we could easily obtain the two defining properties of this gravastar model: the surface energy density $\Sigma$ and surface tension $\Theta$. The junction conditions read [38]

$$
\left[\left[K_{i j}\right]\right]=8 \pi\left[\left[S_{i j}-\gamma_{i j} \frac{S}{2}\right]\right],
$$

where the symbol $[[\ldots]]$ gives the "jump" in a given quantity across the spherical shell (or $r=a$ ), i.e.

$$
[[A]] \equiv A\left(a_{+}\right)-A\left(a_{-}\right) .
$$

The indices $i$ and $j$ correspond to coordinates $t, \theta$, and $\varphi$ which parametrize curves tangential to the spherical shell, $K_{i j}=-\nabla_{i} n_{j}$ is the extrinsic curvature, $n_{\alpha}=$ $(0,1,0,0) / \sqrt{g^{r r}}$ is the unit normal vector, and $S_{i j}$ is the surface stress-energy tensor

$$
S_{i j}=(\Sigma-\Theta) u_{i} u_{j}-\Theta \gamma_{i j}
$$

where $u^{\alpha}=\sqrt{-1 / g_{t t}}(1,0,0,0)$ (or $\vec{u}=\sqrt{-1 / g_{t t}} \vec{\partial}_{t}$ ) is the four-velocity of mass elements on the shell and $\gamma_{\alpha \beta}=$ $g_{\alpha \beta}-n_{\alpha} n_{\beta}$ is the induced three-metric on the shell. We then have

$$
S_{i j}-\gamma_{i j} \frac{S}{2}=(\Sigma-\Theta) u_{i} u_{j}+\frac{\Sigma}{2} \gamma_{i j} .
$$

In the static, spherically symmetric case,

$$
\left[\left[K_{i j}\right]\right]=\left[\frac{\sqrt{g^{r r}}}{2} g_{i j, r}\right] .
$$

Discontinuities in the metric coefficients are then related to the surface energy and surface tension as [35]

$$
[[\sqrt{h}]]=-4 \pi a \Sigma, \quad\left[\left[\frac{f^{\prime} \sqrt{h}}{f}\right]\right]=8 \pi(\Sigma-2 \Theta) .
$$

In order to summarize the above relations and reveal the independent parameter space of a thin-shell gravastar we define

$$
M_{v} \equiv \frac{4 \pi \rho a^{3}}{3}, \quad M_{s} \equiv 4 \pi a^{2} \Sigma,
$$

which would be the volume- and surface-energy contents of the gravastar. In terms of $M_{v}, M_{s}$, and $a$, we can obviously solve for $\rho, \Sigma$, and in addition we have

$$
\begin{gathered}
M=M_{v}+M_{s} \sqrt{1-\frac{2 M_{v}}{a}}+\frac{M_{s}^{2}}{2 a}, \\
\alpha=\frac{1-2 M / a}{1-2 M_{v} / a},
\end{gathered}
$$




$$
\Theta=\frac{1}{8 \pi a}\left[\frac{1-4 M_{v} / a}{\sqrt{1-2 M_{v} / a}}-\frac{1-M / a}{\sqrt{1-2 M / a}}\right] .
$$

As a consequence, gravastar types can be specified by the dimensionless parameters $M_{v} / a$ and $M_{s} / a$. In this paper we only consider a simplified version of the original Mazur-Mottola gravastar, which has vanishing surface en$\operatorname{ergy}(\Sigma=0)$ and

$$
M=M_{v}, \quad 8 \pi a \Theta=-\frac{3 M / a}{\sqrt{1-2 M / a}} .
$$

However it is convenient to keep our notation general enough, because nonradial oscillations of a gravastar will in general produce nonzero variations of the surface energy (i.e., $\delta \Sigma \neq 0$ ).

\section{GRAVITATIONAL PERTURBATIONS}

In both the interior (de Sitter) and exterior (Schwarzschild) background space-times we consider perturbations in the Regge-Wheeler (RW) gauge [39], writing

$$
d s^{2}=d s_{0}^{2}+\left(\delta_{\mathrm{RW}} g_{\mu \nu}\right) d x^{\mu} d x^{\nu}
$$

with

$$
\left\|\delta_{\mathrm{RW}} g_{\mu \nu}\right\|=\left[\begin{array}{cccc}
f(r) H_{0}(t, r) Y_{l m} & H_{1}(t, r) Y_{l m} & -h_{0}(t, r) \frac{1}{\sin \theta} \frac{\partial Y_{l m}}{\partial \varphi} & h_{0}(t, r) \sin \theta \frac{\partial Y_{l m}}{\partial \theta} \\
* & \frac{H_{2}(t, r) Y_{l m}}{h(r)} & -h_{1}(t, r) \frac{1}{\sin \theta} \frac{\partial Y_{l m}}{\partial \varphi} & h_{1}(t, r) \sin \theta \frac{\partial Y_{l m}}{\partial \theta} \\
* & * & r^{2} K(t, r) Y_{l m} & 0 \\
* & * & * & r^{2} \sin ^{2} \theta K(t, r) Y_{l m}
\end{array}\right],
$$

rameter $C \equiv(2 M / a)^{3}$, related to the parameter $\mu=M / a$ of Ref. [36] by $C=8 \mu^{3}$. Then we have (assuming $\Sigma=0$ )

$$
f(r)=1-\frac{8 \pi \rho}{3} r^{2}=1-\frac{2 M}{a^{3}} r^{2} \equiv 1-C(r / 2 M)^{2} .
$$

perturbations. The linearized Einstein equations automatically require $H_{0}=H_{2} \equiv H$.

In the rest of this section we work out perturbations of the gravastar space-time, including the dynamics of the shell itself, in three steps. In Sec. III A, we present the wellknown solution of the perturbation equations in de Sitter space-time in terms of hypergeometric functions and choose the solution that is regular at the origin $(r=0)$. In Sec. III B we review metric perturbations in the Schwarzschild exterior. Finally, in Sec. III C we work out the junction conditions relating the interior and exterior RW perturbations.

\section{A. The de Sitter interior}

The usual way to obtain the interior solution for perturbed stars is by direct integration of the system of ordinary differential equations (ODEs) [40-42]. Integrating a regular solution from the center would give boundary conditions at the stellar radius (or, in the case of a gravastar, at the location of the shell). For the de Sitter interior $(r<a)$ no numerical integrations are required, because a regular solution of the perturbation equations can be obtained in terms of hypergeometric functions $[4,43]$. To establish notation we review the basic equations below. Let us express the metric in terms of a compactness pa-
In the de Sitter interior both axial and polar perturbations can be reduced to the study of the (frequency-domain) master equation

$$
\frac{d^{2} \Psi^{\text {in }}}{d r_{*}^{2}}+\left[\omega^{2}-V_{\text {in }}(r)\right] \Psi^{\text {in }}=0, \quad r<a,
$$

where

$$
V_{\text {in }}(r)=\frac{l(l+1)}{r^{2}} f(r)
$$

and we introduced the tortoise coordinate, defined as usual by the condition $d r / d r_{*}=f(r)$, which in this case yields

$$
r_{*}=\sqrt{\frac{3}{8 \pi \rho}} \operatorname{arctanh}\left[\left(\frac{8 \pi \rho r^{2}}{3}\right)^{1 / 2}\right], \quad r<a .
$$

In terms of $r$, the master equation reads

$$
\frac{\partial^{2} \Psi^{\mathrm{in}}}{\partial r^{2}}+\frac{f^{\prime}}{f} \frac{\partial \Psi^{\mathrm{in}}}{\partial r}+\frac{\omega^{2}-V_{\text {in }}(r)}{f^{2}} \Psi^{\mathrm{in}}=0,
$$

where a prime denotes a derivative with respect to $r$. Near the origin, solutions of Eq. (3.7) behave as $A r^{l+1}+B r^{-l}$. By requiring regularity at the center $(r=0)$ we get, up to a multiplicative constant,

$$
\Psi^{\text {in }}=r^{l+1}\left(1-C(r / 2 M)^{2}\right)^{-i(M \omega / \sqrt{C})} F\left[\frac{l+2-i \frac{2 M \omega}{\sqrt{C}}}{2}, \frac{1+l-i \frac{2 M \omega}{\sqrt{C}}}{2}, l+\frac{3}{2}, \frac{C r^{2}}{4 M^{2}}\right]
$$


where $F(a, b, c, z)$ is the hypergeometric function [44]. From $\Psi^{\text {in }}$ and its derivative we can get the axial perturbation variables in the frequency domain [45]:

$$
h_{1}=\frac{r}{f} \Psi^{\mathrm{in}}, \quad h_{0}=-\frac{i}{\omega} \frac{d}{d r_{*}}\left(r \Psi^{\mathrm{in}}\right) .
$$

The polar metric functions $K$ and $H_{1}$ can be obtained immediately from

$$
\begin{aligned}
& K=\frac{l(l+1)}{2 r} \Psi^{\mathrm{in}}+\frac{d \Psi^{\mathrm{in}}}{d r_{*}}, \\
& H_{1}=\frac{i \omega r}{f}\left(\frac{\Psi^{\mathrm{in}}}{r}+\frac{d \Psi^{\mathrm{in}}}{d r_{*}}\right) .
\end{aligned}
$$

The quantity $H\left(=H_{0}=H_{2}\right)$ and its derivatives can then be found from the algebraic relation

$$
\begin{aligned}
{\left[\frac{l(l+1)}{2}-\frac{1}{f}-\frac{\omega^{2} r^{2}}{f}\right] K } & +\left[-i \omega r-i \frac{l(l+1) C r}{8 M^{2} \omega}\right] H_{1} \\
& -\frac{(l-1)(l+2)}{2} H=0 .
\end{aligned}
$$

This procedure fixes all metric quantities and their derivatives in the interior. Here and henceforth in the paper we drop the dependence of $h_{0}, h_{1}, H, H_{1}$, and $K$ on $\omega$ and $r$.

\section{B. The Schwarzschild exterior}

In the Schwarzschild exterior, axial and polar perturbations obey different master equations [46]. The determination of the axial perturbation variables can still be reduced to the solution of the RW equation [39], a Schrödinger-like ODE identical to Eq. (3.7):

$$
\frac{\partial^{2} \Psi^{\text {out }}}{\partial r^{2}}+\frac{f^{\prime}}{f} \frac{\partial \Psi^{\text {out }}}{\partial r}+\frac{\omega^{2}-V_{\text {out }}(r)}{f^{2}} \Psi^{\text {out }}=0,
$$

where

$$
V_{\text {out }}=f\left(\frac{l(l+1)}{r^{2}}-\frac{6 M}{r^{3}}\right) .
$$

The metric can then be obtained from Eqs. (3.9), with $f(r)$ given by Eq. (2.2).

The perturbed Einstein equations relate the polar variables $\left(K, H, H_{1}\right)$ via three differential equations:

$$
\begin{gathered}
\frac{d}{d r}\left(f H_{1}\right)-i \omega(H+K)=0, \\
-i \omega H_{1}+f\left(H^{\prime}-K^{\prime}\right)+f^{\prime} H=0, \\
K^{\prime}-\frac{H}{r}+\left[\frac{1}{r}-\frac{f^{\prime}}{2 f}\right] K+i \frac{l(l+1)}{2 \omega r^{2}} H_{1}=0,
\end{gathered}
$$

and an algebraic relation:

$$
\begin{array}{r}
{\left[\frac{l(l+1)}{2}-1+\frac{r f^{\prime}}{2}\left(1-\frac{r f^{\prime}}{2 f}\right)-\frac{\omega^{2} r^{2}}{f}\right] K} \\
+\left[-i \omega r+i \frac{l(l+1)}{4 \omega} f^{\prime}\right] H_{1}-\left[\frac{l(l+1)}{2}-f+\frac{r f^{\prime}}{2}\right] H \\
=0 .
\end{array}
$$

Note that if we make the appropriate choice for $f(r)$, Eqs. (3.15), (3.16), and (3.17) and the algebraic relation also apply to the interior de Sitter spacetime [cf. Equation (3.12)].

The Zerilli function $Z^{\text {out }}(r)$ [45], which satisfies a wave equation, and its spatial derivative are also constructed from $H_{1}$ and $K$ as

$$
Z^{\text {out }}=\frac{H_{1}^{\text {out }}-A_{3} K^{\text {out }}}{A_{2}-A_{1} A_{3}}
$$

$$
\frac{d Z^{\text {out }}}{d r_{*}}=\frac{A_{2} K^{\text {out }}-A_{1} H_{1}^{\text {out }}}{A_{2}-A_{1} A_{3}},
$$

where

$$
\begin{gathered}
A_{1}=\frac{6 M^{2}+\lambda / 2(1+\lambda / 2) r^{2}+3 / 2 \lambda M r}{r^{2}(3 M+r \lambda / 2)}, \\
A_{2}=\frac{i \omega\left(-3 M^{2}-3 / 2 \lambda M r+r^{2} \lambda / 2\right)}{r(3 M+r \lambda / 2) f}, \\
A_{3}=i \omega \frac{r}{f},
\end{gathered}
$$

and $\lambda=(l-1)(l+2)$. The metric perturbations can then be obtained by integrating the Zerilli equation outward, starting from $r=a_{+}$. It is also useful to recall that (in the exterior) we can switch from the Zerilli function $Z^{\text {out }}(r)$ (3.20) to the RW function $\Psi^{\text {out }}(r)$ by using a differential relation between polar and axial variables discussed in Chandrasekhar's book [46]:

$\alpha_{-} \Psi^{\text {out }}=\eta Z^{\text {out }}-\frac{d Z^{\text {out }}}{d r_{*}}, \quad \frac{d \Psi^{\text {out }}}{d r_{*}}=\alpha_{+} Z^{\text {out }}-\eta \Psi^{\text {out }}$,

where

$\alpha_{ \pm}=\frac{\lambda(\lambda+2)}{12 M} \pm i \omega, \quad \eta=\frac{\lambda(\lambda+2)}{12 M}+\frac{6 M f(r)}{r(\lambda r+6 M)}$.

From $Z^{\text {out }}$ and $d Z^{\text {out }} / d r_{*}$ we can easily compute $\Psi^{\text {out }}$ and $d \Psi^{\text {out }} / d r_{*}$ outside the shell and use them as initial conditions to integrate the RW equation outward. Leins et al. [47] showed that this procedure is convenient to compute polar oscillation modes by the continued fraction method; more details on the QNM calculation are given in Appendix B. 


\section{The matching conditions}

In this section we discuss the most delicate part of the perturbation problem, namely, the junction conditions for the RW perturbation variables across the shell. Here we only outline our strategy and present the results; more details are given in Appendix A. Technically, the application of Israel's junction conditions is easier if the shell's world tube happens to coincide with a fixed coordinate sphere at constant radius. However this is incompatible with choosing the RW gauge in both the interior and the exterior, which is convenient to cast the perturbation equations into simple forms. In fact, such a choice of gauge does not leave any freedom. We must explicitly parametrize the three-dimensional motion of each mass element on the shell and then perform the matching on a moving shell. In order to take advantage of both the simplicity of the field equations and the convenience of matching on a fixed coordinate sphere, we carry out the matching in the following way. We first construct a particular coordinate transformation (for both the exterior and interior spacetimes) such that in the new coordinate system, any mass on the shell remains static on the coordinate sphere with $r=$ $a$. In this new coordinate system the metric perturbations will no longer be RW, but will be augmented by quantities that carry information about how masses on the shell move in the RW gauge. The stress-energy tensor of masses on the shell will correspondingly be modified. We then carry out the matching at $r=a$ and obtain junction conditions relating the interior and exterior metric perturbations, plus equations of motion for matter on the shell. As could be anticipated from the general features of oscillations of nonrotating stars, axial perturbations do not couple to matter motion and the axial junction conditions are very simple, basically imposing continuity of the master variable $\Psi$ and of its first derivative. Polar perturbations, on the other hand, do couple to matter motion, so polar junction conditions do involve the shell dynamics, i.e. its equation of state.

We parametrize the world line of matter elements on the shell in terms of the coordinates $(t, r, \theta, \varphi)$ as follows:

$$
\begin{array}{cc}
t=\tau / \sqrt{f(a)}+\delta t\left(\tau, \theta_{*}, \varphi_{*}\right), & r=a+\delta r\left(\tau, \theta_{*}, \varphi_{*}\right), \\
\theta=\theta_{*}+\delta \theta\left(\tau, \theta_{*}, \varphi_{*}\right), \quad \varphi=\varphi_{*}+\delta \varphi\left(\tau, \theta_{*}, \varphi_{*}\right),
\end{array}
$$

where $\theta_{*}$ and $\varphi_{*}$ identify physical mass elements on the sphere, while $\tau$ parametrizes their proper time. Note that the Lagrangian equations of motion will not be the same for the interior and exterior space-times. Therefore, points with the same $t, \theta$, and $\varphi$ coordinates are not in general the same when viewed from the interior and from the exterior. As shown in Appendix A, the four-velocity of the mass element $\left(\theta_{*}, \varphi_{*}\right)$ at the scaled proper time $\hat{t} \equiv \tau / \sqrt{f(a)}$ is, to leading order in the perturbation variables,

$$
u^{\alpha}=[f(a)]^{-1 / 2}(1+\delta \dot{t}, \delta \dot{r}, \delta \dot{\theta}, \delta \dot{\varphi}) .
$$

We now carry out a gauge transformation which maps the shell to a fixed location (note that two different gauge transformations are required for the exterior and for the interior). For any general gauge transformation $\bar{x}^{\bar{\alpha}}=x^{\alpha}-$ $\xi^{\alpha}\left(x^{\mu}\right)$ we have, to first order in $\xi^{\mu}$,

$$
\delta g_{\alpha \beta}=\bar{g}_{\alpha \beta}\left(\bar{x}^{\mu}\right)-g_{\alpha \beta}\left(\bar{x}^{\mu}\right)=\xi_{\beta ; \alpha}\left(\bar{x}^{\mu}\right)+\xi_{\alpha ; \beta}\left(\bar{x}^{\mu}\right),
$$

where the semicolon represents a covariant derivative with respect to the four-metric and $\bar{g}_{\alpha \beta}\left(\bar{x}^{\mu}\right)$ is the metric tensor in the new coordinate system. We impose that, when evaluated at $(t, r, \theta, \varphi)=\left(\hat{t}, a, \theta_{*}, \varphi_{*}\right)$, the vector $\xi^{\alpha}$ coincides with $(\delta t, \delta r, \delta \theta, \delta \varphi)$, so that in the new coordinate system we will have

$$
\bar{t}=\tau / \sqrt{f(a)}, \quad \bar{r}=a, \quad \bar{\theta}=\theta_{*}, \quad \bar{\varphi}=\varphi_{*},
$$

where we are ignoring second-order corrections. The full metric in the new coordinate system is

$$
\bar{g}_{\alpha \beta}=g_{\alpha \beta}^{(0)}+\delta_{\mathrm{RW}} g_{\alpha \beta}+\delta g_{\alpha \beta},
$$

where $g_{\alpha \beta}^{(0)}$ is the static gravastar background metric, given by Eq. (2.1). The explicit form of $\xi^{\mu}$ and the corresponding changes in the metric components, Eq. (3.26), are given in Appendix A, where the equations of motion, as well as the gauge transformation, are presented systematically in a multipole expansion. We then match components of $\bar{g}_{\alpha \beta}$ along the shell, which now simply sits at $\left(\hat{t}, a, \theta_{*}, \varphi_{*}\right)$, and apply Israel's junction conditions to the extrinsic curvature given by $\bar{g}_{\alpha \beta}$. For axial perturbations these matching conditions read

$$
\left[\left[h_{0}\right]\right]=0, \quad\left[\left[\sqrt{h} h_{1}\right]\right]=0 .
$$

For thin shell gravastars $h(r)$ is continuous across the shell, implying continuity of the RW function $\Psi$ and its derivative $\Psi^{\prime}$ across the shell [cf. Equation (3.9)]. In more general cases where $h(r)$ may have a discontinuity across the shell the axial junction conditions (3.29) show that $\Psi$ must also be discontinuous.

The treatment of polar perturbations is more involved and it yields the following relations, determining the jump of the polar metric functions at the shell:

$$
\begin{gathered}
{[[K]]=0,} \\
{\left[\left[K^{\prime}\right]\right]=-8 \pi \frac{\delta \Sigma}{\sqrt{f(a)}},}
\end{gathered}
$$




$$
\begin{aligned}
& \frac{2 M}{a^{2}}[[H]]-\left[\left[H f^{\prime}\right]\right]-2 f(a)\left[\left[H^{\prime}\right]\right]+4 i \omega\left[\left[H_{1}\right]\right] \\
& \quad=16 \pi \sqrt{f(a)}\left(1+2 v_{s}^{2}\right) \delta \Sigma .
\end{aligned}
$$

Note that $f(r)$ is continuous across the shell [cf. Equation (2.3)]. The parameter $v_{s}$ in the equations above depends on the equation of state on the thin shell, $\Theta=\Theta(\Sigma)$ :

$$
v_{s}^{2} \equiv-\left(\frac{\partial \Theta}{\partial \Sigma}\right)_{\Sigma=0}
$$

and it has the dimensions of a velocity. One might naively interpret $v_{s}$ as the speed of sound on the thin shell and require $v_{s} \leq 1$, i.e. that the speed of sound cannot exceed the speed of light. Furthermore, for a shell of ordinary, stable matter we would have $v_{s}^{2}>0$. The standard argument used to deduce that $v_{s}^{2}>0$ does not necessarily hold when one deals with exotic matter (as in the case of gravastars and wormholes), so the specification of upper and lower bounds on $v_{s}$ may require a detailed microphysical model of the exotic matter itself $[48,49]$. In our discussion of gravastar stability we will consider the whole range of $v_{s}$, but we will primarily focus on the range $0 \leq$ $v_{s}^{2} \leq 1$. The application of the polar junction conditions is more involved than the axial case due to their complexity, which arises from the fact that polar perturbations couple to oscillations of the shell.

Here we note that even though we have three quantities $\left(K, H, H_{1}\right)$ that satisfy a coupled system of first-order ODEs in both the interior and exterior, in each region there is an algebraic relation relating the three quantities. For this reason we only need to impose two independent junction conditions, and Eqs. (3.30), (3.31), and (3.32) provide exactly two independent relations among $K, H$, and $H_{1}$ (after eliminating $\delta \Sigma$ ). Alternatively, the number of junction conditions can be obtained considering that all metric perturbations can be expressed in terms of $\Psi^{\text {in }}$ and $\Psi^{\text {out }}$, and that each of these master variables satisfies a secondorder ODE. More specifically, we use Eqs. (3.30), (3.31), and (3.32) to determine two relations among $\left(\Psi^{\mathrm{in}}, \partial_{r} \Psi^{\mathrm{in}}\right)$ and $\left(\Psi^{\text {out }}, \partial_{r} \Psi^{\text {out }}\right)$, plus the corresponding $\delta \Sigma$.

\section{NUMERICAL RESULTS}

Some axial and polar QNM frequencies for a static thinshell gravastar, as computed by the continued fraction method, are plotted in Figs. 1 and 2. The C++ code used for the calculations is an update of the FORTRAN code used in Ref. [50] to verify and extend results on stellar oscillations by Kokkotas [51] and Leins, Nollert, and Soffel [47]. For axial modes, our numerical results are in excellent agreement with the thin-shell limit of the QNM frequencies computed by Chirenti and Rezzolla [36] and with Fiziev's calculation of the axial QNMs of ultracompact objects with a totally reflecting surface (compare Figs. 3 and 4 of Ref. [37]).

To find the QNM frequencies we adopt the following numerical procedure. We usually fix $\mu=0.4$ and (for polar perturbations) we choose a constant value of $v_{s}$. In the calculations leading to Figs. 1 and 2 we chose, somewhat arbitrarily, $v_{s}^{2}=0.1$. Later in this section we will discuss the dependence of polar modes on $v_{s}$.

As explained in Appendix B, within the continued fraction method the complex QNM frequencies can be determined as the roots of any of the $n$ equations $f_{n}(\omega)=0$ [cf. Equation (B19)], where $n$ is the "inversion index" of the continued fraction. To locate QNMs we first fix a value of $\mu$ (usually $\mu=0.4$ ). We compute the real and imagi-
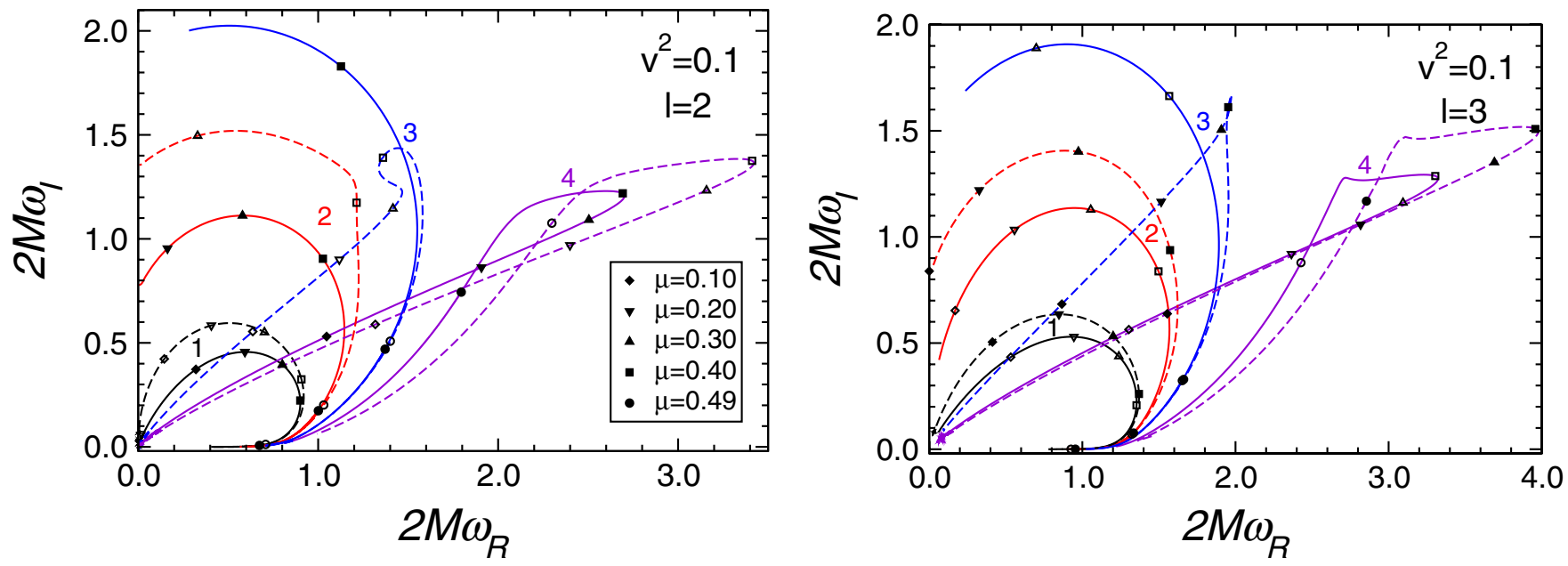

FIG. 1 (color online). First few axial (continuous lines) and polar (dashed lines) QNMs of a thin-shell gravastar with $v_{s}^{2}=0.1$. In the left panel we follow modes with $l=2$ as the compactness $\mu$ varies. In the right panel we do the same for modes with $l=3$. Along each track we mark by different symbols (as indicated in the legend) the points where $\mu=0.1,0.2,0.3,0.4$, and 0.49 . Our numerical method becomes less reliable when $2 M \omega_{I}$ is large and when the modes approach the pure-imaginary axis. Numbers next to the polar and axial modes refer to the overtone index $N(N=1$ being the fundamental mode). 

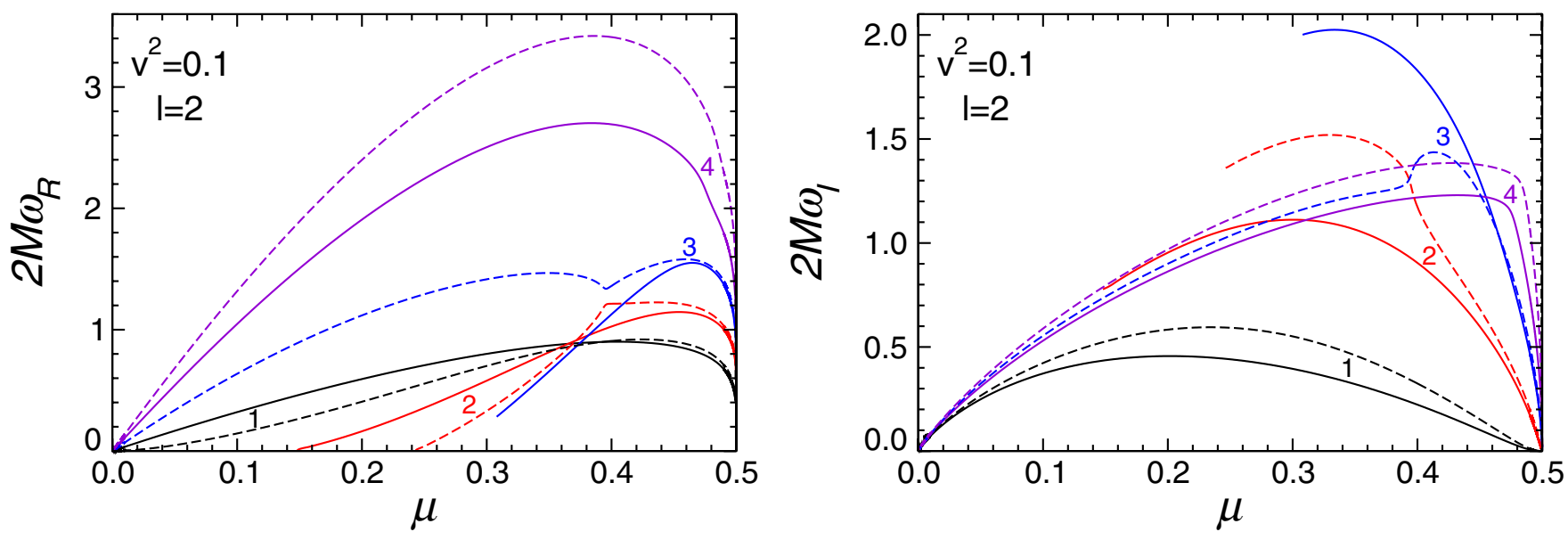

FIG. 2 (color online). Real (left) and imaginary (right) part of polar and axial QNMs with $l=2$ as functions of $\mu$ for $v_{s}^{2}=0.1$. Line styles are the same as in Fig. 1. Numbers refer to the overtone index.

nary parts of $f_{n}(\omega)$ for a given inversion index $n$ on a suitably chosen grid of $\left(\omega_{R}, \omega_{I}\right)$ values, and make contour plots of the curves along which the two functions are zero. The intersections of these curves are used as initial guesses for the quasinormal frequencies; more precise values are then obtained using Müller's method [52]. For fixed $n$ (say, $n=0$ ) this method singles out some spurious roots besides the physical QNM frequencies. The spurious roots can easily be ruled out, since they are not present for different values of $n$. Looking for roots with $n=0$ is usually sufficient, but sometimes we get more stable numerical solutions for $n=1$ and $n=2$ when the QNMs have a large imaginary part ( $\omega_{I} \gtrsim 1.5$ or so).

The QNM spectrum with $\mu=0.4$ corresponds to the empty (polar) and filled (axial) squares in the left panel of Fig. 1, respectively. Starting from $\mu=0.4$, we follow each QNM as $\mu \rightarrow 0$ and as $\mu \rightarrow 1 / 2$ to produce the tracks displayed in the figure. For this value of $v_{s}$ some of these tracks end at the origin in the limit $\mu \rightarrow 0$, while others hit the pure-imaginary axis at some finite limiting compactness $\mu_{\text {imag }}$ (e.g. $\mu_{\text {imag }} \simeq 0.24$ for the first polar mode). The mode frequencies usually move clockwise in the complex plane (with the exception of QNMs displaying "loops") as $\mu$ is increased. The imaginary part of both axial modes (continuous lines) and polar modes (dashed lines) becomes very small as $\mu \rightarrow 1 / 2$, i.e. when the gravastar most closely approximates a $\mathrm{BH}$. The behavior is perhaps clearer from Fig. 2, where we separately show the real and imaginary parts as functions of $\mu$.

For both axial and polar spectra the dependence of the mode frequencies on the gravastar compactness resembles that of "ordinary" ultracompact stars: see e.g. Fig. 3 of Ref. [53]. Intuitive models that capture most of the physics of this problem have been presented in Refs. [54,55]. In their terminology, modes that emerge from the origin in Fig. 1 when $\mu \sim 0$ are $w$-modes or curvature modes, roughly corresponding to waves trapped inside the star.
Modes emerging from the imaginary axis at some generally nonvanishing compactness are $w_{\mathrm{II}}$-modes [47] or interface modes, and they are similar in nature to acoustic waves scattered off a hard sphere $[55,56]$. The only qualitative difference with Fig. 3 of Ref. [53] are the loops appearing for higher-order $w$-modes, for which we have no analytical understanding.

The fact that $w$-modes are effectively waves "trapped inside the star", while $w_{\mathrm{II}}$-modes are interface modes, similar to acoustic waves scattered off a hard sphere, is also clear from the behavior of their wave functions in the stellar interior. The real and imaginary parts of the wave functions are shown in Fig. 3 (see Ref. [47] for comparison with the wave functions of ordinary stars). This figure shows eigenfunctions computed at the polar QNM frequencies for the first four $w$-modes and $w_{\mathrm{II}}$-modes with $l=2$ and $v_{s}^{2}=0.1$. The plot shows that $w$-modes can be thought of as standing waves inside the gravastar, and that the overtone number corresponds to the number of nodes in the real and imaginary parts of the eigenfunction. The situation is different for $w_{\mathrm{II}}$-modes, where the wave function has a maximum close to the shell, as expected for interface modes.

One of our most important conclusions is that neither axial nor polar modes of a gravastar reduce to the QNMS of a Schwarzschild $B H$ when $\mu \rightarrow 1 / 2$. In this limit, the real part of most modes is extremely small (much smaller than the Schwarzschild result, $2 M \omega_{R} \simeq 0.74734$ for the fundamental mode with $l=2$ [4]). Indeed, the QNM spectrum is drastically different from the QNM spectrum of a Schwarzschild BH: when $\mu \rightarrow 1 / 2$ the entire spectrum seems to collapse toward the origin. This is in sharp contrast with the Schwarzschild $\mathrm{BH}$ case and, as first noted in Ref. [36], it can be used to discern a very compact gravastar from a BH. In Appendix $\mathrm{C}$ we prove analytically that the QNM frequencies of a gravastar do not reduce to those of a Schwarzschild BH as $\mu \rightarrow 1 / 2$. The proof is based on the 

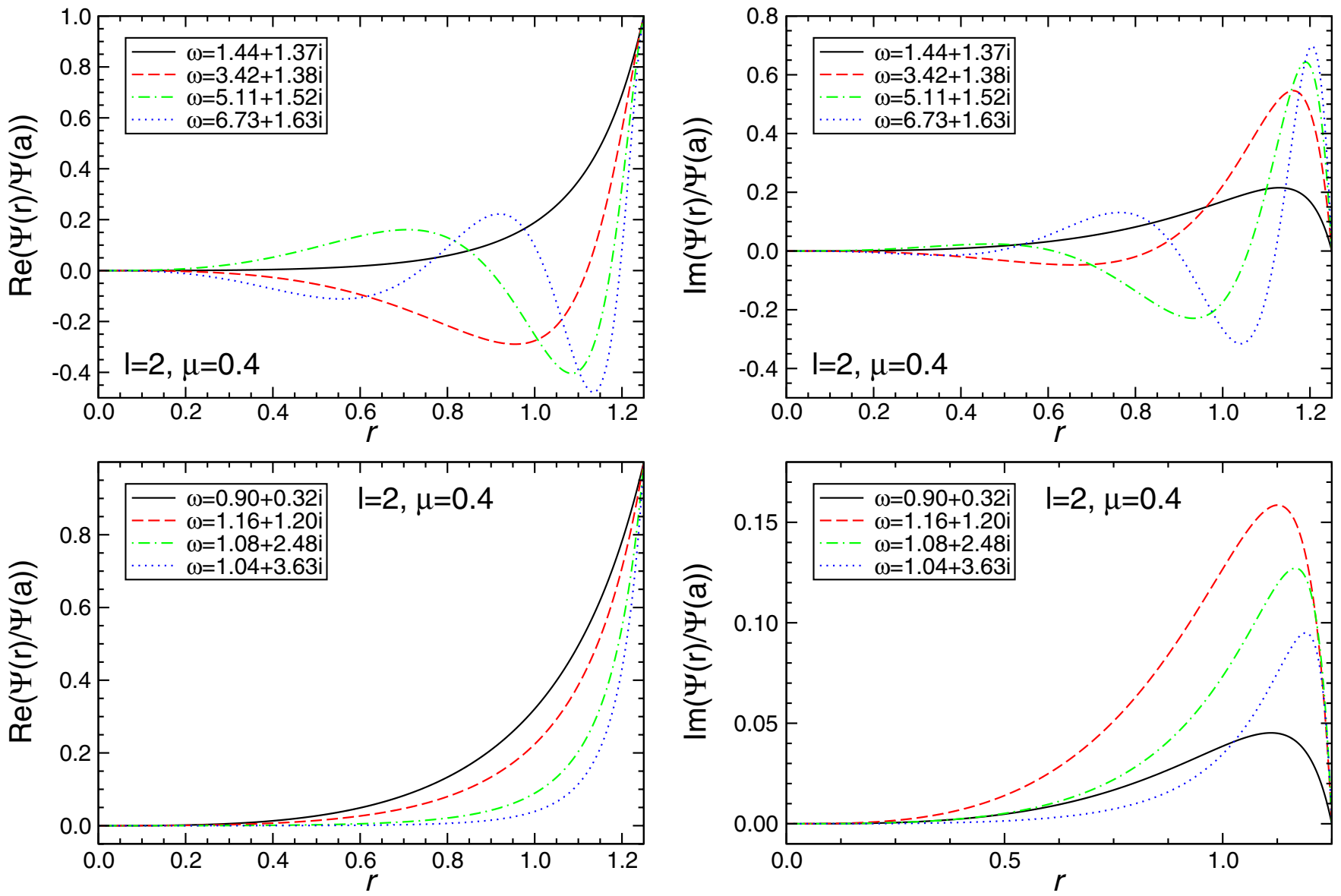

FIG. 3 (color online). Top row: real and imaginary part of the wave function in the interior for the first four $w$-modes. Bottom row: real and imaginary part of the wave function in the interior for the first four $w_{\mathrm{II}}$-modes. In both cases we consider polar QNMs with $l=2$ and $v_{s}^{2}=0.1$.

observation that the Zerilli wave function for polar modes is continuous in this limit.

It is clear from the figures that axial and polar modes do not have the same spectra for general values of $\mu$.

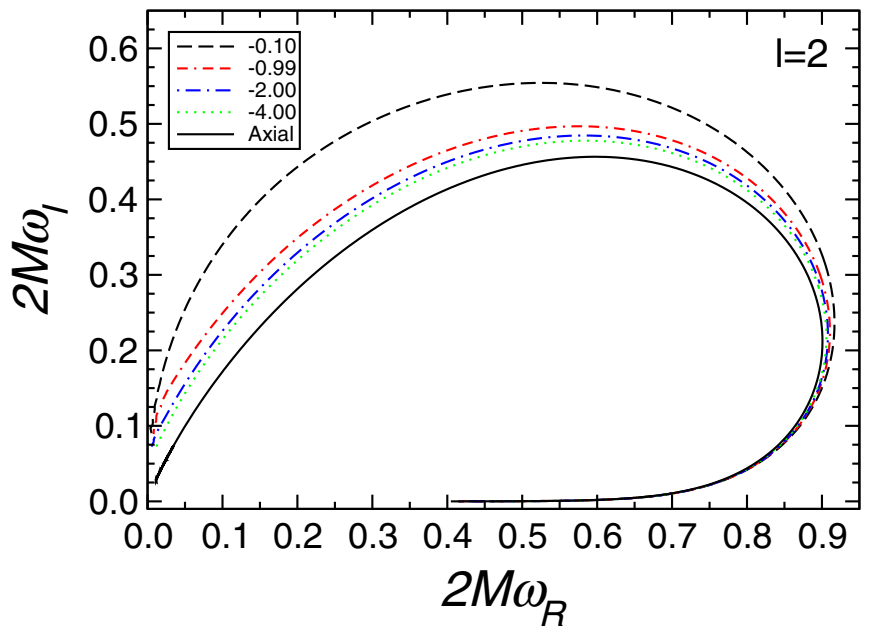

However, Figs. 1, 2, and 4 provide evidence that axial and polar QNMs do become isospectral when the gravastar compactness approaches that of a Schwarzschild BH $(\mu \rightarrow$ $0.5)$. An analytic proof of isospectrality in the high-

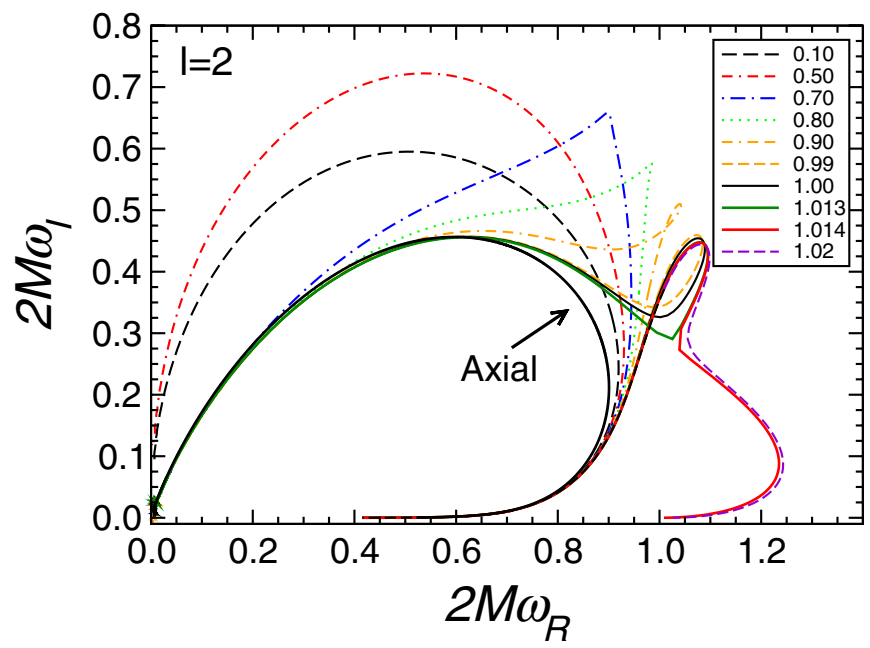

FIG. 4 (color online). Tracks of the fundamental polar and axial $w$-modes for different values of the "sound speed" parameter $v_{s}$ when $v_{s}^{2}<0$ (left) and when $v_{s}^{2}>0$ (right). Different line styles correspond to different values of $v_{s}^{2}$, as indicated in the legend. 

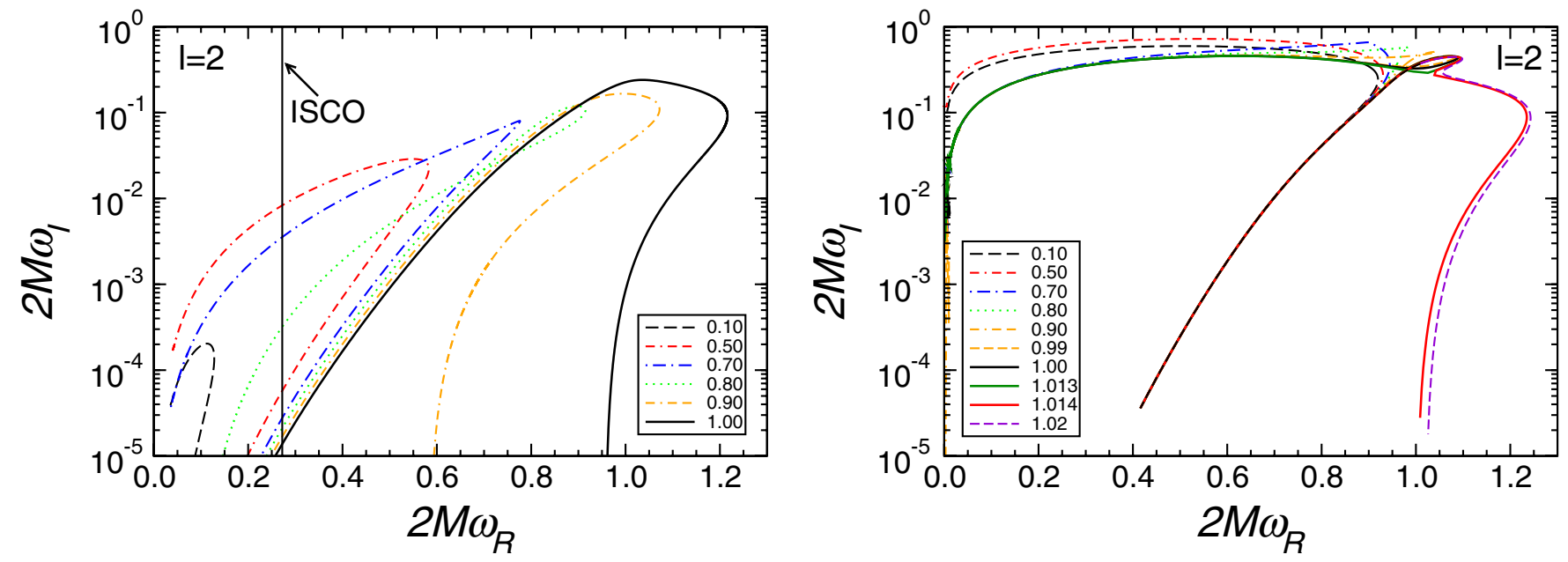

FIG. 5 (color online). Left: spectrum of the weakly damped family of QNMs. The vertical line corresponds to twice the orbital frequency of a particle in circular orbit at the innermost stable circular orbit (ISCO), as we will discuss in a follow-up paper, only QNMs to the left of the line can be excited by a compact object inspiraling into the gravastar along quasicircular orbits. In the case $v_{s}=0.8$ the mode "turns around" describing a loop in the complex plane. For $v_{s} \lesssim 0.8$ the modes move clockwise in the complex plane as $\mu$ increases. For $v_{s} \gtrsim 0.8$ they move counterclockwise and they cross the real axis at finite compactness. To facilitate comparison, in the right panel we show again the right panel of Fig. 4 using a logarithmic scale for the imaginary part.

compactness limit is given in Appendix C, where we show that in this limit both the Zerilli and RW functions are continuous at the shell.

From the matching conditions (3.29), (3.30), (3.31), and (3.32) it is quite clear that polar QNMs (unlike axial QNMs) should depend on $v_{s}$, i.e. [by Eq. (3.33)] on the equation of state on the shell. This is a new feature that does not arise in the case of axial perturbations [36]. The situation closely parallels the ordinary stellar perturbation problem $[42,57]$. The role played by the equation of state in the dynamical stability of gravastars against spherically symmetric perturbations was discussed in Ref. [35]. Our calculations extend the considerations of that paper to nonradial oscillations.

The $v_{s}$-dependence of the modes is studied in Figs. 4-6. In Fig. 4 we show the tracks described in the complex plane by the fundamental polar and axial $w$-mode as we vary the compactness parameter $\mu$. The fundamental axial mode does not depend on the equation of state parameter, as expected, but the polar modes do change as a function of $v_{s}$. The standard argument used to deduce that the speed of sound $v_{s}^{2}>0$ does not necessarily hold when one deals with exotic matter (as in the case of gravastars and wormholes). Therefore, for completeness, in the left panel of
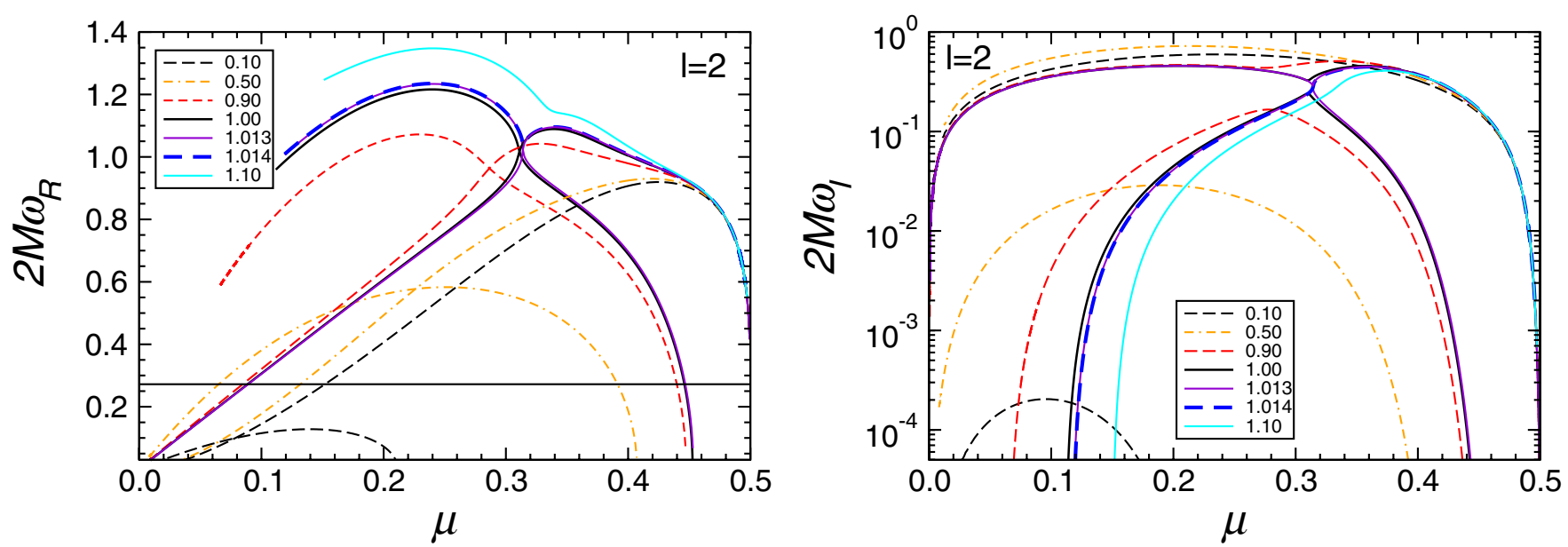

FIG. 6 (color online). Real (left) and imaginary parts (right) of the fundamental polar $w$-mode for different values of the equation of state parameter $v_{s}$. Different line styles correspond to different values of $v_{s}^{2}$, as indicated in the legend. The horizontal line in the left panel corresponds to twice the orbital frequency of a particle in circular orbit at the ISCO: only modes below the line can be excited during a quasicircular inspiral. 
Fig. 4 we compute polar QNMs when $v_{s}^{2}<0$. Different line styles correspond to different values of $v_{s}^{2}$, as indicated in the legend. The solid black line reproduces the fundamental axial $w$-mode of Figs. 1 and 2. The dashed black line corresponds to the fundamental polar $w$-mode for a shell with low sound speed $\left(v_{s}^{2}=0.1\right)$, corresponding to the fundamental polar $w$-mode of Figs. 1 and 2. The dashdash-dotted (red), dash-dotted (blue), and dotted (green) lines represent a marginally subluminal, imaginary sound speed $\left(v_{s}^{2}=-0.99\right)$ and superluminal sound speeds $\left(v_{s}^{2}=\right.$ -2 and $v_{s}^{2}=-4$, respectively). Nothing particularly striking happens in this regime: QNM frequencies for polar and axial perturbations are different in all cases, but for large compactness the results become $v_{s}$-independent and modes of different parity become approximately isospectral, in agreement with the analytical results of Appendix C. Furthermore, as $\left|v_{s}\right| \rightarrow \infty$ the polar modes seem to approach the axial mode. We can perhaps understand this behavior if we think that the shell is effectively becoming so stiff that matter decouples from the spacetime dynamics, and only the "space-time" character of the oscillations survives.

The situation is more interesting in the case $v_{s}^{2}>0$, displayed in the right panel of Fig. 4. At first (when $v_{s}^{2} \leq 0.5$ or so) the modes show a behavior similar to that seen for $v_{s}^{2}<0$, albeit in the opposite direction (i.e. the real and imaginary parts of the QNM frequencies increase rather than decreasing when $\left|v_{s}\right|$ increases). When $v_{s}^{2}=0.7$ a cusp develops, and as the speed of sound approaches the speed of light (for $v_{s}^{2} \gtrsim 0.9$ in the figure) the modes "turn around" describing a loop in the complex plane. The area of this loop in the complex plane increases until the sound speed reaches a critical value $1.013 \leq$ $v_{\text {crit }}^{2} \leq 1.014$ (corresponding to $v_{\text {crit }} \simeq 1.007$ ). For $v_{s}>$ $v_{\text {crit }}$ the QNM behavior changes quite drastically. The complex mode frequencies still approach the $\omega_{I}=0$ axis clockwise as $\mu \rightarrow 0.5$. However, as $\mu$ decreases the modes approach the axis $\omega_{I}=0$ very rapidly along tracks which are now tangent to the lower branch of the fundamental axial mode.

Even more interestingly, when $v_{s}^{2}>0$ there is also a second family of QNMs with a very small imaginary part. A plot of the eigenfunctions shows that these modes are similar in nature to the $w_{\mathrm{II}}$-modes. The trajectories described in the complex plane by some of these weakly damped QNMs are shown in the left panel of Fig. 5. For comparison, the right panel of Fig. 5 shows some of the ordinary modes. These ordinary modes are the same as in the right panel of Fig. 4, except that this time we use a logarithmic scale for the imaginary part. The second family of QNMs plotted in the left panel has very long damping, and in this sense it is similar to the $s$-modes of ordinary ultracompact stars discussed by Chandrasekhar and Ferrari [57]. There is, however, an important difference: unlike the $s$-modes, which exist only when a star is extremely com- pact, the weakly damped modes of a thin-shell gravastar only exist for small compactness $\mu<\mu_{\text {crit }}$.

In the left (right) panel of Fig. 6 we show the real (imaginary) parts of both families of QNMs as functions of $\mu$ for selected values of $v_{s}$. Both the real and imaginary part of the weakly damped modes tend to zero at some finite, $v_{s}$-dependent compactness $\mu$. The range where weakly damped modes exist increases with $v_{s}^{2}$. The fact that both the mode frequency and its damping tend to zero at the critical compactness $\mu_{\text {crit }}$ suggests that the mode somehow "disappears" there, rather than undergoing a nonradial instability, but this conjecture deserves a more careful analytical study. Plots similar to those shown in Fig. 6 show that the imaginary part of ordinary modes with $v_{s}^{2} \gtrsim 0.84\left(v_{s} \geq 0.92\right)$ rapidly approaches zero at some finite compactness $\mu$ while the real part of the modes stays finite. We are unable to track QNMs numerically when $2 M \omega_{I} \lesssim 10^{-5}$ using the continued fraction method, and in any case we cannot really prove by numerical methods that $\omega_{I} \rightarrow 0$ at some finite compactness $\mu>0$. When $v_{s}^{2}=$ 1.0134 the two families of modes approach each other along a cusp, but their frequencies and damping times never cross. The ordinary family of modes exists all the way up to $\mu=0.5$ but it becomes unstable (in the sense that the imaginary part of the mode crosses the real axis with the real part remaining finite) at some finite, $v_{s}$-dependent value of the compactness $\mu$.

Summarizing, our numerical results suggest that (1) weakly damped QNMs only exist when their compactness is smaller than some $v_{s}$-dependent critical threshold and (2) when $v_{s}>0$ and $v_{s}^{2} \geqslant 0.84$, the imaginary part of a QNM crosses the real axis at another critical threshold whose value can be estimated by extrapolation. Nonrotating thin-shell gravastars should be unstable against nonradial perturbations when their compactness is smaller than this critical value.

The two thresholds are plotted in Fig. 7. Weakly damped QNMs with $l=2$ exist only below the dashed line and, according to our extrapolation of numerical results, thinshell gravastars should be unstable to nonradial perturbations with $l=2$ below the solid line (we verified that the instability condition for $l=3$ is less stringent than for $l=$ 2 ). The dashed line extends up to $v_{s}^{2}<1.0134$, where our numerical search for weakly damped QNMs becomes impractical (the modes trace smaller and smaller loops in the complex plane and they seem to disappear when the compactness is still smaller than $\mu=0.5$ ).

To validate results from the continued fraction method we used an independent numerical approach: the resonance method [57-59], which is applicable to QNMs with $\omega_{I} \ll \omega_{R}$. The resonance method was first used by Chandrasekhar and Ferrari in their analysis of gravitational wave scattering by constant-density, ultracompact stars $[57,58]$. Chandrasekhar and Ferrari showed that the radial potential describing odd-parity perturbations of these stars 


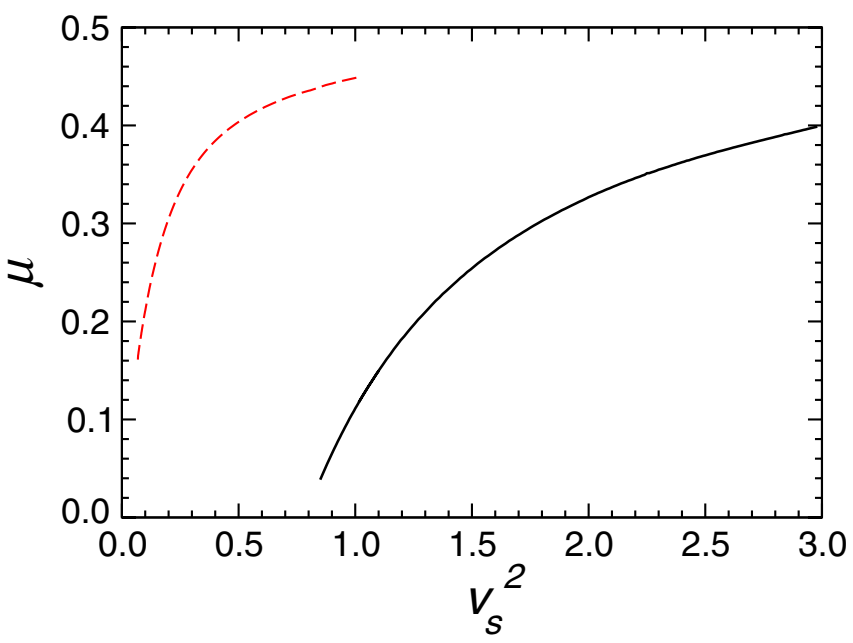

FIG. 7 (color online). Significant thresholds in the $\left(\mu, v_{s}^{2}\right)$ plane. Thin-shell gravastars should be unstable with respect to nonradial perturbations below the solid line, corresponding to ordinary QNM frequencies whose imaginary part crosses the zero axis while their real part stays finite. The dashed line corresponds to the "vanishing point" of weakly damped QNM frequencies, i.e. to the point where both their real and imaginary parts have a zero crossing. We could not find weakly damped modes in the region above the dashed line.

displays a local minimum as well as a maximum when the stellar compactness $\mu \gtrsim 0.39$. If this minimum is sufficiently deep, quasistationary, "trapped" states can exist: gravitational waves can only leak out to infinity by "tunneling" through the potential barrier. The damping time of these modes is very long, so they were dubbed "slowly damped" modes (or $s$-modes) [57].

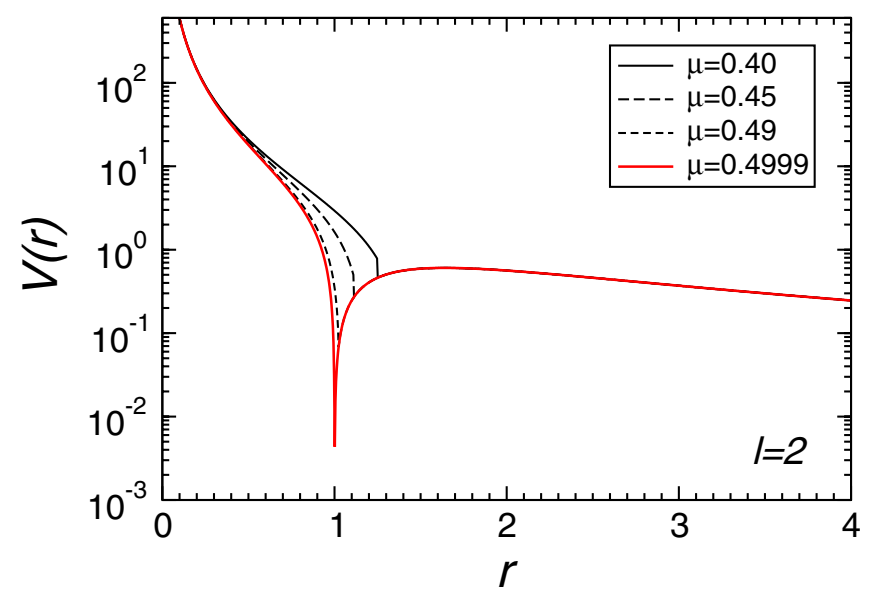

FIG. 8 (color online). The potential governing axial and polar perturbations for different values of the gravastar compactness $\mu \equiv M / a$, where $a$ is the location of the shell (see [57], showing a similar plot for axial perturbations of constant-density stars). The potential develops a minimum when $\mu \gtrsim 0.43$. Note that the polar and axial perturbations in the interior are both governed by the same potential, given in Eq. (3.5) below.
A straightforward analysis of Eq. (3.5) and an inspection of Fig. 8 show that the axial potential for a gravastar develops a minimum when $\mu \gtrsim 0.43$. The compactness of ordinary stars is limited by the Buchdal limit $(\mu<$ $4 / 9 \simeq 0.4444$ ), but since gravastars can be considerably more compact than this limit, $s$-modes can exist all the way down to the "Schwarzschild limit" $(\mu \rightarrow 0.5)$. These modes can be computed via the continued fraction method, but since they are long-lived the resonance method, which is computationally very simple, provides very accurate estimates of their frequencies and damping times. We find that the resonance method and the continued fraction method are in very good agreement whenever the resonance method is applicable. In the limit $\mu \rightarrow 1 / 2$ we have $\operatorname{Im}(\omega) \ll \operatorname{Re}(\omega)$, and all QNM frequencies can be interpreted as trapped states.

The resonance method essentially confirms our continued fraction results for modes with $\omega_{I} \ll \omega_{R}$. In particular, it provides additional numerical evidence for the conjectured nonradial instability of thin-shell gravastars with low compactness. Despite the numerical evidence, an analytic confirmation of our estimates of the instability threshold would be highly desirable.

\section{CONCLUSIONS AND OUTLOOK}

In this work we have studied the nonradial perturbations on nonrotating, thin-shell gravastars. It should not be too hard to extend our formalism to the more complex case of five-layer gravastars of the type originally proposed by Mazur and Mottola (see [36] for a treatment of the axial case).

A presumably less trivial extension concerns rotating gravastars. Slowly rotating gravastars may be unstable against scalar perturbations because of an exponential growth of the perturbations due to superradiance, the socalled "ergoregion instability" [60,61]. An extension of the present formalism can be used to study nonradial gravitational perturbations of slowly rotating gravastars and to discuss their ergoregion instability, which is believed to be stronger for gravitational perturbations [60]. For gravastars this instability is due to superradiant gravitational wave scattering in the ergoregion, so it is essentially the same as the " $w$-mode instability" discussed by Kokkotas et al. for ultracompact stars [62]. The main difference is that gravastars can be more compact than constant-density stars, so we may expect the instability to be stronger.

Finally, our formalism can be applied to explore nonradial gravitational perturbations of nonrotating wormholes, where the position of the throat plays a role similar to the thin shell of a gravastar. Such an analysis could confirm or disprove some conjectures on the similarity of the QNM spectra of wormholes and BHs [63].

In follow-up work we will extend our study to explore QNM excitation by compact objects in closed orbits 
around thin-shell gravastars (see $[64,65]$ for similar studies considering the excitation of axial modes by scattering orbits). According to preliminary estimates by Norte, following the weak-gravity expansion of Poisson and Sasaki [66] (extended by Li and Lovelace [17] to general boundary conditions), the gravitational wave luminosity can change dramatically at the resonances, while being very close to the $\mathrm{BH}$ value in nonresonant conditions. A weakfield study should provide a reasonably accurate estimate of the orbital frequency at which the resonance occurs, but it can only provide a leading-order estimate of the radiated energy. A more precise characterization requires the numerical integration of the perturbation equations [67-70]. In a follow-up paper we will compare gravitational radiation from extreme mass-ratio inspirals in thin-shell gravastar space-times to the $\mathrm{BH}$ results of Refs. [71-74].

\section{ACKNOWLEDGMENTS}

This work was partially supported by FCT-Portugal through projects PTDC/FIS/64175/2006, PTDC/FIS/ 098025/2008, and PTDC/FIS/098032/2008. Y. C. was supported by NSF Grant Nos. PHY-0653653 and PHY0601459, and the David and Barbara Groce Start-up Fund at Caltech. E. B.'s research was supported by NSF Grant No. PHY-0900735.

\section{APPENDIX A: PERTURBATION EQUATIONS AND MATCHING CONDITIONS}

In this appendix we develop the formalism to study polar and axial nonradial (linear) oscillations of an object consisting of a thin spherical shell separating two spherically symmetric regions. Though we are mainly interested in thin-shell gravastars, we shall keep the discussion as general as possible. We focus on a background metric of the form (2.1), keeping $f(r)$ and $h(r)$ generic.

The RW gauge is incompatible with the requirement that the shell's world tube sits at a fixed location. We deal with this issue in two steps: (i) we choose a coordinate system (system A) such that perturbations in both the interior and exterior are in the RW gauge; (ii) we write down the equations of motion of mass elements on the shell in this coordinate system (separately for the interior and the exterior), and try to apply matching conditions on the boundary of this moving shell. We then transform to a new coordinate system (system B) in which the shell is fixed, simplifying the matching procedure. System B is an auxiliary tool for matching: when we consider perturbations of the gravastar induced (say) by orbiting particles we will mostly use system A, which is the usual RW gauge.

Step (i) is a straightforward adaptation of formulas in Sec. III. In the RW gauge, polar perturbations are defined by three functions $K, H$, and $H_{1}$ for the interior and exterior, respectively. These functions satisfy an algebraic relation and two coupled ODEs, which can be used to reduce the problem to a wave equation. This means that we only need two conditions relating these quantities from the two sides. Similarly, for axial perturbations we have $h_{0}$ and $h_{1}$, which satisfy a coupled wave equation, and again we need only two conditions connecting the interior and exterior.

Now we go directly to step (ii), and parametrize the shell position as in Eqs. (3.24). Let us define

$$
\begin{gathered}
\hat{t} \equiv \tau / \sqrt{f(a)}, \\
\dot{F} \equiv(\partial F / \partial \hat{t})=\sqrt{f(a)}(\partial F / \partial \tau) .
\end{gathered}
$$

Note that $\hat{t}$ is simply a rescaling of proper time of the mass element, and that $f(a)$ is common to the interior and exterior, due to the requirement that the induced metric is continuous. In the absence of perturbations, $\hat{t}$ coincides with $t$. Henceforth, we will use $\left(\hat{t}, \theta_{*}, \varphi_{*}\right)$, to parametrize the mass element. As a consequence, the four-velocity $u^{\alpha}$ of the mass element $\left(\theta_{*}, \varphi_{*}\right)$ would be as in Eq. (3.25). Imposing that $g_{\alpha \beta} u^{\alpha} u^{\beta}=-1$, we actually have to require that

$$
\frac{(1+2 \delta \dot{t}) g_{t t}\left(\hat{t}+\delta t, a+\delta r, \theta_{*}+\delta \theta, \varphi_{*}+\delta \varphi\right)}{f(a)}=-1,
$$

which, to leading order, is

$$
\begin{aligned}
f^{\prime}(a) \delta r\left(\hat{t}, \theta_{*}, \varphi_{*}\right)+2 f(a) \delta \dot{t}\left(\hat{t}, \theta_{*}, \varphi_{*}\right) & \\
& -\delta_{\mathrm{RW}} g_{t t}\left(\hat{t}, \theta_{*}, \varphi_{*}\right)=0 .
\end{aligned}
$$

Here $\delta_{\mathrm{RW}} g_{t t}$ is the $t t$-component of the metric perturbation in the RW gauge.

We will now carry out a gauge transformation, both in the exterior and in the interior, which maps the shell to a fixed sphere. As explained in the main text, for any general gauge transformation $\bar{x}^{\bar{\alpha}}=x^{\alpha}-\xi^{\alpha}\left(x^{\mu}\right)$ we have

$$
\begin{aligned}
g_{\alpha \beta}\left(x^{\mu}\right) & =\bar{g}_{\mu \nu}\left(\bar{x}^{\rho}\right)\left(\frac{\partial \bar{x}^{\mu}}{\partial x^{\alpha}}\right)\left(\frac{\partial \bar{x}^{\nu}}{\partial x^{\beta}}\right) \\
& =\left[\bar{g}_{\alpha \beta}-\bar{g}_{\alpha \nu} \xi_{, \beta}^{\nu}-\bar{g}_{\mu \beta} \xi_{, \alpha}^{\mu}\right]_{\bar{x}^{\rho}},
\end{aligned}
$$

where we have ignored terms of second order in $\xi^{\mu}$. Noting that

$$
g_{\alpha \beta}\left(x^{\mu}\right)=g_{\alpha \beta}\left(\bar{x}^{\mu}+\xi^{\mu}\right),
$$

we obtain Eq. (3.26). This is the desired form, because we want to use $\xi^{\mu}$ to transform to a coordinate system $\bar{x}^{\mu}$ where mass elements on the shell are fixed in spatial location and move uniformly in the time direction: i.e., for mass elements on the shell, $\bar{x}^{\mu}=\left(\hat{t}, a, \theta_{*}, \varphi_{*}\right)$. Then Israel's junction conditions will be applied to the RW metric evaluated at a fixed coordinate location $\left(\hat{t}, a, \theta_{*}, \varphi_{*}\right)$, and in terms of the transformation generators 
(which are related to the motion of the shell in the RW gauge).

Let us make four consecutive transformations

$$
\begin{aligned}
& \xi_{(0)}^{\alpha}=\left[-f^{-1}(r) y(t) Y_{l m}, 0,0,0\right], \\
& \xi_{(1)}^{\alpha}=\left[0, h(r) z(t) Y_{l m}, 0,0\right], \\
& \xi_{(2)}^{\alpha}=\left[0,0, \frac{w(t)}{r^{2}} Y_{l m, \theta}, \frac{w(t)}{r^{2} \sin ^{2} \theta} Y_{l m, \varphi}\right], \\
& \xi_{(3)}^{\alpha}=\left[0,0, \frac{x(t)}{r^{2} \sin \theta} Y_{l m, \varphi},-\frac{x(t)}{r^{2} \sin \theta} Y_{l m, \theta}\right],
\end{aligned}
$$

or, by lowering indices,

$$
\begin{aligned}
& \xi_{(0) \alpha}=\left[y(t) Y_{l m}, 0,0,0\right], \quad \xi_{(1) \alpha}=\left[0, z(t) Y_{l m}, 0,0\right], \\
& \xi_{(2) \alpha}=\left[0,0, w(t) Y_{l m \mid \theta}, w(t) Y_{l m \mid \varphi}\right], \\
& \xi_{(3) \alpha}=\left[0,0, x(t) \epsilon_{\theta \varphi} Y_{l m}{ }^{\mid \varphi}, x(t) \epsilon_{\varphi \theta} Y_{l m}{ }^{\mid \theta}\right],
\end{aligned}
$$

where the covariant derivative / is defined with respect to the two-dimensional metric

$$
G_{a b}=d \theta^{2}+\sin ^{2} \theta d \varphi^{2} .
$$

We impose that, when evaluated at $(t, r, \theta, \varphi)=(\hat{t}, a$, $\left.\theta_{*}, \varphi_{*}\right)$, the vector $\xi^{\alpha}$ coincides with $(\delta t, \delta r, \delta \theta, \delta \varphi)$, so that in the new coordinate system Eqs. (3.27) will be valid. Such a transformation leads to the following changes in the metric components:

$$
\begin{gathered}
\delta_{(0)} g_{\mu \nu}=\left[\begin{array}{cccc}
2 \dot{y} & -\frac{f^{\prime}}{f} y & y \partial_{\theta} & y \partial_{\varphi} \\
* & & & \\
* & & &
\end{array}\right] Y_{l m}, \\
\delta_{(1)} g_{\mu \nu}=\left[\begin{array}{cccc}
-f^{\prime} h z & \dot{z} & & \\
* & \frac{h^{\prime}}{h} z & z \partial_{\theta} & z \partial_{\varphi} \\
& * & 2 r h z & \\
& * & & 2 r h z \sin ^{2} \theta
\end{array}\right] Y_{l m},
\end{gathered}
$$

which are purely polar perturbations,

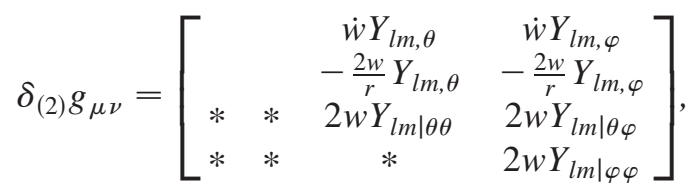

which are also polar perturbations, and finally

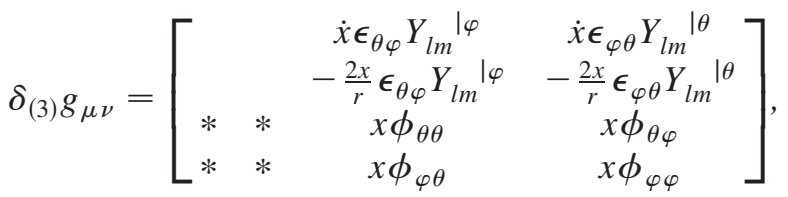

which are axial perturbations. Here we have defined

$$
\phi_{m n}=\epsilon_{m}{ }^{a} Y_{l m \mid n a}+\epsilon_{n}{ }^{a} Y_{l m \mid m a},
$$

where $m, n$, and $a$ run through $\theta$ and $\varphi$. Here $\epsilon_{a b}$ is again defined with respect to the metric (A7), so that

$$
\epsilon_{\theta \varphi}=-\epsilon_{\varphi \theta}=\sin \theta .
$$

In terms of $y$ and $z$, the normalization of the fourvelocity would be written as

$$
\begin{aligned}
0 & =-f^{\prime}(a) h(a) z(\hat{t})+f(a) H(\hat{t}, a)-2 f(a)\left[-f^{-1}(a) \dot{y}(\hat{t})\right] \\
& =-f^{\prime}(a) h(a) z(\hat{t})+f(a) H(\hat{t}, a)+2 \dot{y}(\hat{t}) .
\end{aligned}
$$

Here we note again that $f_{+}=f_{-}$. In this new coordinate system the metric is given by Eq. (3.28), where

$$
\delta g_{\alpha \beta}=\delta_{(1)} g_{\alpha \beta}+\delta_{(2)} g_{\alpha \beta}+\delta_{(3)} g_{\alpha \beta} .
$$

We are now in a position to match metric components along the shell, which simply sits at $\left(\hat{t}, a, \theta_{*}, \varphi_{*}\right)$. Of course, all of the matching conditions will be expressed in terms of the RW metric perturbations, and the motion of the shell in the (internal and external) RW gauges. In the $\theta$ and $\varphi$ directions, we have

$$
\begin{aligned}
x_{+}(\hat{t}) & =x_{-}(\hat{t}), \\
w_{+}(\hat{t}) & =w_{-}(\hat{t}), \\
2 h_{+}(a) z_{+}(\hat{t})+a K_{+}(\hat{t}, a) & =2 h_{-}(\hat{t}) z_{-}(\hat{t})+a K_{-}(\hat{t}, a) .
\end{aligned}
$$

In the $t \theta$ and $t \varphi$ directions we have, in addition,

$$
y_{+}(\hat{t})=y_{-}(\hat{t}), \quad h_{0+}(\hat{t}, a)=h_{0-}(\hat{t}, a),
$$

while in the $t t$ direction the matching condition is automatically satisfied, with

$$
\bar{g}_{t t}\left(\hat{t}, a, \theta_{*}, \varphi_{*}\right)=-f(a),
$$

accurate up to first order in the perturbations. This is a consistency check, since we have imposed that the fourvelocity of mass elements on the shell is

$$
u^{\alpha}=(1 / \sqrt{f(a)}, 0,0,0),
$$

which should have a norm of -1 . In simplified form, we have

$$
\begin{aligned}
{[[x]] } & =[[w]]=[[y]]=\left[\left[h_{0}\right]\right]=[[2 h z+a K]] \\
& =\left[\left[2 \dot{y}+f H-f^{\prime} h z\right]\right]=0 .
\end{aligned}
$$

The symbol [[...]], as defined by Eq. (2.5), gives the jump of any given quantity across the shell.

The four-velocity of mass elements on the shell is given by Eq. (A19). The surface stress-energy tensor of the shell is

$$
\begin{aligned}
S_{j k}= & {\left[\Sigma-\Theta+(\delta \Sigma-\delta \Theta) Y_{l m}\right] u_{j} u_{k} } \\
& -\left[\Theta+\delta \Theta Y_{l m}\right] \gamma_{j k},
\end{aligned}
$$

where $j$ and $k$ go through $t, \theta$ and $\varphi$. Now let us try to 
evaluate the extrinsic curvature of the shell at the location $\left(\hat{t}, a, \theta_{*}, \varphi_{*}\right)$. First of all, we note that

$$
n_{\alpha}=(0,1,0,0) /{\sqrt{\bar{g}^{r r}}}
$$

and the extrinsic curvature is

$$
K_{i j}=-\nabla_{i} n_{j}=-n_{j, i}+\Gamma^{\mu}{ }_{j i} n_{\mu},
$$

where $i, j$ run through $t, \theta, \varphi$. Since $n_{\alpha}$ only has a nonzero $r$-component the first term vanishes, and we obtain

$$
K_{i j}=\frac{1}{\sqrt{g}^{r r}} \Gamma^{r}{ }_{i j} .
$$

From the static configuration of the star, it is easy to obtain that

$$
\begin{aligned}
& \left\|S_{j k}\right\|=\left[\begin{array}{lll}
\Sigma f(a) & & \\
& -a^{2} \Theta & \\
& & -a^{2} \Theta \sin ^{2} \theta
\end{array}\right], \\
&
\end{aligned}
$$

where $j, k=t, \theta, \varphi$. From this we have relations (2.9) for static gravastars.

Now let us focus on first-order quantities. The tensors $S_{j k}$ and $K_{j k}$ can each be decomposed into six terms, for example,

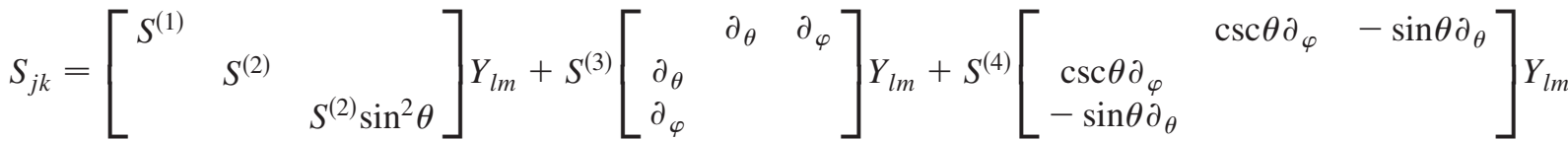

$$
\begin{aligned}
& +S^{(5)}\left[\begin{array}{ccc}
0 & & \\
& Y_{\mid \theta \theta} & Y_{\mid \theta \varphi} \\
& Y_{\mid \varphi \theta} & Y_{\mid \varphi \varphi}
\end{array}\right]+S^{(6)}\left[\begin{array}{lll}
0 & & \\
& \phi_{\theta \theta} & \phi_{\theta \varphi} \\
& \phi_{\varphi \theta} & \phi_{\varphi \varphi}
\end{array}\right]
\end{aligned}
$$

We have

$$
\begin{gathered}
S^{(1)}=f \delta \Sigma+\left[(\Theta-2 \Sigma)\left(f H-h z f^{\prime}+2 \dot{y}\right)\right], \\
S^{(2)}=-a(a K \Theta+2 h z \Theta+a \delta \Theta), \\
S^{(3)}=-\Sigma(y+\dot{w}), \\
S^{(4)}=\Sigma\left(h_{0}-\dot{x}\right), \\
S^{(5)}=-2 \Theta w, \\
S^{(6)}=-\Theta x,
\end{gathered}
$$

and

$$
\begin{aligned}
\bar{K}^{(1)}= & \frac{f \sqrt{h}}{a}\left(H-a K^{\prime}\right)+f \sqrt{h} z \frac{\lambda_{L}+2 h-a h^{\prime}}{a^{2}}, \text { (A29) } \\
\bar{K}^{(2)}= & \frac{a^{2} \sqrt{h}}{2}\left\{K^{\prime}-H^{\prime}+\frac{2\left(\dot{H}_{1}+\ddot{z}\right)}{f}+\left(1+\frac{a f^{\prime}}{2 f}\right) \frac{2 K-H}{a}\right. \\
& +\left[\frac{2\left(h-\lambda_{L}\right)}{a^{2}}+\frac{2 f^{\prime} h+f h^{\prime}}{a f}\right. \\
& \left.\left.+\frac{f f^{\prime} h^{\prime}-2\left(f^{\prime}\right)^{2} h+2 h f f^{\prime \prime}}{2 f^{2}}\right] z\right\}, \\
\bar{K}^{(3)} & =\frac{\sqrt{h}}{a}\left[\frac{a H_{1}}{2}+2 y+a \dot{z}+\left(1+\frac{a f^{\prime}}{2 f}\right) \dot{w}\right], \quad \text { (A330) }
\end{aligned}
$$

$$
\begin{gathered}
\bar{K}^{(4)}=\sqrt{h}\left[\frac{\dot{x}-2 h_{0}}{a}\left(1+\frac{a f^{\prime}}{2 f}\right)+\frac{h_{0}^{\prime}-\dot{h}_{1}}{2}\right], \\
\bar{K}^{(5)}=\frac{2 \sqrt{h}}{a}\left[\frac{a z}{2}+w\left(1+\frac{a f^{\prime}}{2 f}\right)\right], \\
\bar{K}^{(6)}=\sqrt{h}\left[-\frac{h_{1}}{2}+\frac{x}{a}\left(1+\frac{a f^{\prime}}{2 f}\right)\right],
\end{gathered}
$$

where $\lambda_{L}=-l(l+1)$. Here $\bar{K}^{(4)}$ and $\bar{K}^{(6)}$ are axial quantities. The junction condition on $\bar{K}^{(6)}$ yields

$$
\left[\left[\sqrt{h} h_{1}\right]\right]=0,
$$

which together with $\left[\left[h_{0}\right]\right]=0$ completes the junction conditions for axial perturbations (the junction condition on $\bar{K}^{(4)}$ yields an equation of motion for the variable $x$ ). For polar quantities, from $\left[\left[\bar{K}^{(5)}\right]\right]=8 \pi S^{(5)}$ we have

$$
[[\sqrt{h} z]]=0 .
$$

Then matching $\left[\left[\bar{K}^{(3)}\right]\right]=8 \pi S^{(3)}$ gives us an equation of motion for $w$, while matching $\left[\left[\bar{K}^{(1,2)}\right]\right]=8 \pi S^{(1,2)}$ yields

$$
\left[\left[\sqrt{h}\left(\frac{H}{a}-K^{\prime}\right)\right]\right]+\left[\left[\frac{2 h}{a^{2}}-\frac{h^{\prime}}{a}\right]\right] \sqrt{h} z=8 \pi \delta \Sigma,
$$




$$
\begin{gathered}
{\left[\left[\sqrt{h}\left(K^{\prime}-H^{\prime}+\frac{2 \dot{H}_{1}}{f}\right)\right]\right]-\left[\left[\sqrt{h}\left(1+\frac{a f^{\prime}}{2 f}\right) \frac{H}{a}\right]\right]} \\
+\left[\left[\frac{h^{\prime}}{a}-\frac{2 h}{a^{2}}+\frac{f^{\prime \prime} h}{f}-\frac{f^{\prime} h^{\prime}}{2 f}\right]\right] \sqrt{h} z=-16 \pi \delta \Theta .
\end{gathered}
$$

The remaining equations are

$$
\begin{gathered}
{[[K]]=-2[[\sqrt{h}]] \sqrt{h} z / a=8 \pi \Sigma \sqrt{h} z,} \\
{[[H]]=\left[\left[\frac{f^{\prime} \sqrt{h}}{f}\right]\right] \sqrt{h} z=8 \pi(\Sigma-2 \Theta) \sqrt{h} z,} \\
\delta \Theta=-v^{2} \delta \Sigma,
\end{gathered}
$$

where $v$ is defined as in Eq. (3.33).

The formalism described above is more general than we need for a static thin-shell gravastar. It can easily be adapted to more general horizonless space-times and to static wormholes. For the Mazur-Mottolla gravastar, we have

$$
\begin{gathered}
\Sigma=0, \quad \Theta=-\frac{\left[\left[f^{\prime}\right]\right]}{16 \pi \sqrt{f(a)}}, \quad[[f]]=0=\left[\left[f^{\prime \prime}\right]\right], \\
{\left[\left[f^{\prime}\right]\right]=\frac{6 M}{a^{2}}, \quad\left[\left[f^{\prime 2}\right]\right]=-\frac{12 M^{2}}{a^{4}} .}
\end{gathered}
$$

Using the equations of Sec. III A above together with these junction conditions we obtain continuity conditions for the shell position, $[[x]]=[[y]]=[[w]]=[[z]]=0$, and the matching conditions for the axial and polar perturbations functions presented in the main text [Eqs. (3.29), (3.30), (3.31), and (3.32)].

\section{APPENDIX B: THE CONTINUED FRACTION METHOD}

Our numerical search for the QNMs of gravastars is based on the continued fraction method, as modified in $[47,50]$. The QNMs of an oscillating gravastar are solutions of Eqs. (3.7) and (3.13) satisfying the boundary conditions imposed by physical requirements: $\Psi$ should be regular at the origin, have the behavior of a purely outgoing wave at infinity, and satisfy the junction conditions discussed in Sec. IIIC. The QNM frequencies are the (complex) frequencies $\omega=\omega_{R}+i \omega_{R}$ for which these requirements are satisfied.

The numerical determination of the QNM frequencies is nontrivial, especially for modes with large imaginary parts (strongly damped modes). The reason is simple to understand. Solutions of Eq. (3.13) representing outgoing and ingoing waves at infinity have the asymptotic behavior $\Psi^{\text {out }} \sim e^{r_{*} / \tau}$ and $\Psi^{\text {in }} \sim e^{-r_{*} / \tau}$ as $r_{*} \rightarrow \infty$, where $\tau=$ $1 / \omega_{I}$ is the damping time. Therefore, identifying by numerical integration the purely outgoing solutions (that is, those solutions for which $\Psi^{\text {in }}$ is zero) becomes increasingly difficult as the damping of the mode increases. The same problem occurs also in the case of QNMs of BHs, and was solved by Leaver [75]. Leaver found a continued fraction relation that can be regarded as an implicit equation which identifies the quasinormal frequencies, thus circumventing the need to perform an integration out to large values of $r_{*}$. This method was subsequently adapted to the polar and axial oscillations of a star [47,50]. The RW equation, which describes the perturbed space-time outside the gravastar, becomes

$$
\frac{d^{2} \Psi}{d r_{*}^{2}}+\left[\omega^{2}-V_{\text {out }}\right] \Psi=0,
$$

with

$$
V_{\text {out }}=\left(1-\frac{2 M}{r}\right)\left(\frac{l(l+1)}{r^{2}}-\frac{6 M}{r^{3}}\right)
$$

and the tortoise coordinate $r_{*}=r+2 M \ln (r / 2 M-1)$. We shall now write the solution of the RW equation in a power-series form as follows. Defining $z \equiv 1-R_{2} / r$, where $r=R_{2}$ is some point outside the shell of the gravastar, and introducing a function $\phi(z)$, related to $\Psi(r)$ by

$$
\Psi(r)=(r-2 M)^{-i 2 M \omega} e^{-i \omega r} \phi(z) \equiv \chi(r) \phi(z),
$$

one finds that $\phi$ satisfies the differential equation

$$
\begin{aligned}
\left(c_{0}+c_{1} z+c_{2} z^{2}+c_{3} z^{3}\right) \frac{d^{2} \phi}{d z^{2}} & +\left(d_{0}+d_{1} z+d_{2} z^{2}\right) \frac{d \phi}{d z} \\
& +\left(e_{0}+e_{1} z\right) \phi=0 .
\end{aligned}
$$

The constants depend only on $\omega, l$, and $R_{2}$ through the relations

$$
\begin{gathered}
c_{0}=1-\frac{2 M}{R_{2}}, \quad c_{1}=\frac{6 M}{R_{2}}-2, \quad c_{2}=1-\frac{6 M}{R_{2}}, \\
c_{3}=\frac{2 M}{R_{2}}, \quad d_{0}=-2 i \omega R_{2}+\frac{6 M}{R_{2}}-2, \\
d_{1}=2\left(1-\frac{6 M}{R_{2}}\right), \quad d_{2}=\frac{6 M}{R_{2}}, \\
e_{0}=\frac{6 M}{R_{2}}-l(l+1), \quad e_{1}=-\frac{6 M}{R_{2}} .
\end{gathered}
$$

Let us now perform a power-series expansion of $\phi(z)$ :

$$
\phi(z)=\sum_{n=0}^{\infty} a_{n} z^{n} .
$$

By substituting this expression in Eq. (B4), the expansion coefficients $a_{n}$ are found to satisfy a four-term recurrence relation of the form

$$
\begin{aligned}
& \alpha_{1} a_{2}+\beta_{1} a_{1}+\gamma_{1} a_{0}=0, \quad n=1, \\
& \alpha_{n} a_{n+1}+\beta_{n} a_{n}+\gamma_{n} a_{n-1}+\delta_{n} a_{n-2}=0, \quad n \geq 2,
\end{aligned}
$$

where 
$\alpha_{n}=n(n+1) c_{0}, \quad n \geq 1$,

$\beta_{n}=(n-1) n c_{1}+n d_{0}, \quad n \geq 1$,

$\gamma_{n}=(n-2)(n-1) c_{2}+(n-1) d_{1}+e_{0}, \quad n \geq 1$,

$\delta_{n}=(n-3)(n-2) c_{3}+(n-2) d_{2}+e_{1}, \quad n \geq 2$.

(B7)

The coefficient $a_{0}$ is a normalization constant, and it is irrelevant from the point of view of imposing outgoingwave boundary conditions. The ratio $a_{1} / a_{0}$ can simply be determined by imposing the continuity of $\Psi$ and $\Psi^{\prime}$ at $r=$ $R_{2}$, since from Eq. (B3) it follows that

$$
\begin{gathered}
a_{0}=\left.\phi\right|_{z=0}=\frac{\Psi\left(R_{2}\right)}{\chi\left(R_{2}\right)}, \\
\frac{a_{1}}{a_{0}}=\frac{R_{2}}{\Psi\left(R_{2}\right)}\left[\Psi^{\prime}\left(R_{2}\right)+\frac{i \omega R_{2}}{R_{2}-2 M} \Psi\left(R_{2}\right)\right] .
\end{gathered}
$$

In the axial case, the values of $\Psi\left(R_{2}\right)$ and $\Psi^{\prime}\left(R_{2}\right)$ can be obtained by the taking the interior solution (3.8) at $r=a_{-}$ and applying the junction conditions (3.29) to determine the wave function in the exterior, i.e. at $r=a_{+}$. From then onward, we can numerically integrate the RW equation (3.13) up to $r=R_{2}$. The remaining coefficients can then be determined by recursion from Eq. (B6). In the polar case we proceed in a similar way: we obtain the Zerilli function $Z^{\text {out }}$ and its derivative at $r=a_{+}$by imposing the matching conditions (3.30), (3.31), and (3.32). Then we use Eq. (3.24) to obtain the corresponding RW function at $r=$ $a_{+}$, integrate forward to find $a_{0}$ and $a_{1}$, and finally obtain the remaining coefficients by recursion.

To apply the continued fraction technique, it is easier to consider three-term recurrence relations. Leaver has shown that the four-term recurrence relation (B6) can be reduced to a three-term recurrence relation by a Gaussian elimination step [76]. Define

$$
\hat{\beta}_{0}=\frac{a_{1}}{a_{0}}, \quad \hat{\alpha}_{0}=-1,
$$

where $a_{1} / a_{0}$ is obtained numerically from Eq. (B9). Now set

$$
\begin{array}{ll}
\hat{\alpha}_{n}=\alpha_{n}, & \hat{\beta}_{n}=\beta_{n}, \quad(n=0,1), \\
\hat{\gamma}_{n}=\gamma_{n}, & (n=1),
\end{array}
$$

and for $n \geq 2$

$$
\begin{gathered}
\hat{\alpha}_{n}=\alpha_{n}, \quad \hat{\beta}_{n}=\beta_{n}-\frac{\hat{\alpha}_{n-1} \delta_{n}}{\hat{\gamma}_{n-1}}, \\
\hat{\gamma}_{n}=\gamma_{n}-\frac{\hat{\beta}_{n-1} \delta_{n}}{\hat{\gamma}_{n-1}}, \quad \hat{\delta}_{n}=0 .
\end{gathered}
$$

By this Gaussian elimination, Eq. (B6) reduces to

$$
\hat{\alpha}_{n} a_{n+1}+\hat{\beta}_{n} a_{n}+\hat{\gamma}_{n} a_{n-1}=0 .
$$

The elimination step is not as trivial as it may seem, because in the process one of the three independent solutions to Eq. (B6) is lost. It can be shown that this solution is not relevant for our purposes [47].

We now turn to investigating the asymptotic behavior of the coefficients $a_{n}$ in the expansion (B5). Let us make the ansatz

$$
\lim _{n \rightarrow \infty} \frac{a_{n+1}}{a_{n}}=1+\frac{h}{n^{1 / 2}}+\frac{k}{n}+\cdots
$$

Dividing Eq. (B6) by $n^{2} a_{n}$, keeping terms up to $\sim n^{-3 / 2}$, and equating to zero the various terms in the expansion in powers of $n^{-1 / 2}$ we find the relations

$$
\begin{gathered}
c_{0}+c_{1}+c_{2}+c_{3}=0, \quad 2 c_{0}+c_{1}-c_{3}=0, \\
h^{2}=2 i \omega R_{2}, \quad k=-\frac{3}{4}+i \omega\left(R_{2}+2 M\right) .
\end{gathered}
$$

The first two of these equations are identities. Substituting the second pair of equations in Eq. (B14) we get

$$
\lim _{n \rightarrow \infty} a_{n}=n^{-3 / 4+i \omega\left(R_{2}+2 M\right)} e^{ \pm 2 \sqrt{2 i \omega R_{2} n}} .
$$

According to a definition given by Gautschi [77], the solution of Eq. (B14) corresponding to the plus sign in Eq. (B16) is said to be dominant, whereas that corresponding to the minus sign is said to be minimal [77]. If we select the minimal solution the expansion (B5) is absolutely and uniformly convergent outside the star, provided that we choose $R_{2}$ such that $R_{2} / 2<a<R_{2}$ and $R_{2}>2$. Furthermore, according to Eq. (B3), the solution to Eq. (B1) behaves as a pure outgoing wave at infinity, i.e. it is the QNM wave function. Thus, the key point is to identify the minimal solutions of Eq. (B14). According to a theorem due to Pincherle [77], if Eq. (B14) has a minimal solution then the following continued fraction relation holds:

$$
\frac{a_{1}}{a_{0}}=\frac{-\hat{\gamma}_{1}}{\hat{\beta}_{1}-} \frac{\hat{\alpha}_{1} \hat{\gamma}_{2}}{\hat{\beta}_{2}-} \frac{\hat{\alpha}_{2} \hat{\gamma}_{3}}{\hat{\beta}_{3}-} \ldots,
$$

where the continued fraction on the right-hand side is convergent and completely determined since the coefficients $\hat{\alpha}_{n}, \hat{\beta}_{n}$, and $\hat{\gamma}_{n}$, defined in Eqs. (B11) and (B12) are known functions of $\omega$. Moreover, from Eqs. (B9) and (B10) it is apparent that the dependence on the stellar model is all contained in the ratio $a_{1} / a_{0}$. Keeping in mind the definitions (B10), Eq. (B17) can be recast in the form

$$
0=f_{0}(\omega)=\hat{\beta}_{0}-\frac{\hat{\alpha}_{0} \hat{\gamma}_{1}}{\hat{\beta}_{1}-} \frac{\hat{\alpha}_{1}}{\hat{\beta}_{2}-} \frac{\hat{\gamma}_{2}}{\hat{\beta}_{3}-} \ldots
$$

Using the inversion properties of continued fractions [78], the latter equation can be inverted $n$ times to yield: 


$$
\begin{aligned}
0= & f_{n}(\omega) \\
= & \hat{\beta}_{n}-\frac{\hat{\alpha}_{n-1} \hat{\gamma}_{n}}{\hat{\beta}_{n-1}-} \frac{\hat{\alpha}_{n-2} \hat{\gamma}_{n-1}}{\hat{\beta}_{n-2}-} \ldots \frac{\hat{\alpha}_{0} \hat{\gamma}_{1}}{\hat{\beta}_{0}}-\frac{\hat{\alpha}_{n} \hat{\gamma}_{n+1}}{\hat{\beta}_{n+1}-} \\
& \times \frac{\hat{\alpha}_{n+1} \hat{\gamma}_{n+2}}{\hat{\beta}_{n+2}-} \frac{\hat{\alpha}_{n+2} \hat{\gamma}_{n+3}}{\hat{\beta}_{n+3}-} \ldots
\end{aligned}
$$

for $n=1,2, \ldots$. These $n$ conditions are analytically equivalent to Eq. (B18). However, since the functions $f_{n}(\omega)$ have different convergence properties, each of them is best suited to find the quasinormal frequencies in a given region of the complex $\omega$ plane. This is the main reason for the accuracy and flexibility of the continued fraction technique.

\section{APPENDIX C: HIGH-COMPACTNESS LIMIT}

To investigate the behavior at the surface of the gravastar in the high-compactness limit, we use the $z \rightarrow 1-z$ transformation law for the hypergeometric function [44],

$$
\begin{aligned}
F(a, b, c, z)= & (1-z)^{c-a-b} \frac{\Gamma(c) \Gamma(a+b-c)}{\Gamma(a) \Gamma(b)} \\
& \times F(c-a, c-b, c-a-b+1,1-z) \\
& +\frac{\Gamma(c) \Gamma(c-a-b)}{\Gamma(c-a) \Gamma(c-b)} \\
& \times F(a, b,-c+a+b+1,1-z) .
\end{aligned}
$$

Using Eq. (3.8) in the limit when $C \rightarrow 1$ and $r \rightarrow a$ we get

$$
\begin{aligned}
\Psi \approx & {\left[\frac{2(a-r)}{a}\right]^{i M \omega} \frac{\Gamma\left(l+\frac{3}{2}\right) \Gamma(-i 2 M \omega)}{\Gamma\left(\frac{2+l-i 2 M \omega}{2}\right) \Gamma\left(\frac{1+l-i 2 M \omega}{2}\right)} } \\
& +\left[\frac{2(a-r)}{a}\right]^{-i M \omega} \frac{\Gamma\left(l+\frac{3}{2}\right) \Gamma(i 2 M \omega)}{\Gamma\left(\frac{1+l+i 2 M \omega}{2}\right) \Gamma\left(\frac{2+l+i 2 M \omega}{2}\right)} .
\end{aligned}
$$

Within our conventions the first term is ingoing, while the second term is outgoing near the surface. So it is clear that in this regime both in- and outgoing modes are present, and QNMs do not reduce to the Schwarzschild QNMs (which require only ingoing waves). Furthermore, we can clearly see that ingoing and outgoing waves always have the same magnitude: the gravastar appears like a reflecting object, as suggested by [79]. Because this reflection happens in a polar coordinate system, it can simply be interpreted as due to the fact that waves going into a lossless gravastar will reemerge without loss. Nevertheless, such a behavior already supports the conclusions of Ref. [60,80], which showed that (for scalar fields) the ergoregion instability is more effective when the surfaces of the compact objects behave like a "perfect mirror" in this sense.

It is easy to show that in the high-compactness limit

$$
\Psi^{\prime}\left(a_{-}\right)=\frac{i \omega a}{a-2 M} \Psi\left(a_{-}\right) .
$$

Solving for the metric quantities we find, up to dominant terms in $a-2 M$,

$$
\begin{gathered}
K\left(a_{-}\right)=\frac{l(l+1)+2 i a \omega}{2 a} \Psi\left(a_{-}\right), \\
K^{\prime}\left(a_{-}\right)=-\omega \frac{-i l(l+1)+2 \omega a}{2(a-2 M)} \Psi\left(a_{-}\right), \\
H_{1}\left(a_{-}\right)=-\frac{\omega a(\omega a-i)}{a-2 M} \Psi\left(a_{-}\right), \\
H_{1}^{\prime}\left(a_{-}\right)=-\frac{\omega(\omega a-i)\left(4 M+i \omega a^{2}\right)}{(a-2 M)^{2}} \Psi\left(a_{-}\right), \\
H_{0}\left(a_{-}\right)=-\frac{\omega a(\omega a-i)}{a-2 M} \Psi\left(a_{-}\right), \\
H_{0}^{\prime}\left(a_{-}\right)=-\frac{\omega(\omega a-2 i)\left(2 M+i \omega a^{2}\right)}{(a-2 M)^{2}} \Psi\left(a_{-}\right) .
\end{gathered}
$$

In the exterior we get

$$
\begin{gathered}
K\left(a_{+}\right)=\frac{l(l+1)+2 i a \omega}{2 a} \Psi\left(a_{-}\right), \\
K^{\prime}\left(a_{+}\right)=-\omega \frac{-i l(l+1)+4 \omega a}{2(a-2 M)} \Psi\left(a_{-}\right), \\
H_{1}\left(a_{+}\right)=-\frac{\omega M(i+4 M \omega)}{a-2 M} \Psi\left(a_{-}\right), \\
H_{1}^{\prime}\left(a_{+}\right)=-\frac{M \omega(2 M \omega+i)(1-4 i M \omega)}{(a-2 M)^{2}} \Psi\left(a_{-}\right), \\
H_{0}\left(a_{+}\right)=-\frac{M \omega(i+4 M \omega)}{a-2 M} \Psi\left(a_{-}\right), \\
H_{0}^{\prime}\left(a_{+}\right)=H_{1}^{\prime}\left(a_{+}\right) .
\end{gathered}
$$

Notice that, even though $H_{1}$ is not continuous at $r=a$, the Zerilli function is. Indeed, we get

$$
Z^{\text {out }}\left(a_{+}\right)=\Psi\left(a_{-}\right), \quad \Psi\left(a_{-}\right)=\Psi\left(a_{+}\right) .
$$

Thus, we conclude that in the high-compactness limit, the master wave function for polar perturbations is continuous across the shell. A trivial extension of the known Schwarzschild results then shows that polar and axial perturbations are isospectral for large compactness, i.e. when $a \rightarrow 2 M$ and $\mu \rightarrow 1 / 2$. 
[1] F. Melia, arXiv:0705.1537.

[2] R. Narayan, New J. Phys. 7, 199 (2005).

[3] A. Mueller, Proc. Sci. P2GC (2006) 017 [arXiv:astro-ph/ 0701228].

[4] E. Berti, V. Cardoso, and A. O. Starinets, Classical Quantum Gravity 26, 163001 (2009).

[5] A. M. Ghez et al., Astrophys. J. 689, 1044 (2008).

[6] S. Doeleman et al., Nature (London) 455, 78 (2008).

[7] R. Schodel et al., Nature (London) 419, 694 (2002).

[8] E. Maoz, Astrophys. J. 494, L181 (1998).

[9] M. Coleman Miller, Mon. Not. R. Astron. Soc. Lett. 367, L32 (2006).

[10] A. E. Broderick, A. Loeb, and R. Narayan, Astrophys. J. 701, 1357 (2009).

[11] D. Psaltis, arXiv:0806.1531.

[12] M. A. Abramowicz, W. Kluzniak, and J.-P. Lasota, Astron. Astrophys. 396, L31 (2002).

[13] W. Kundt, Gen. Relativ. Gravit. 41, 1967 (2009).

[14] M. Visser, arXiv:0901.4365.

[15] F. D. Ryan, Phys. Rev. D 52, 5707 (1995).

[16] F. D. Ryan, Phys. Rev. D 56, 1845 (1997).

[17] C. Li and G. Lovelace, Phys. Rev. D 77, 064022 (2008).

[18] N. A. Collins and S. A. Hughes, Phys. Rev. D 69, 124022 (2004).

[19] K. Glampedakis and S. Babak, Classical Quantum Gravity 23, 4167 (2006).

[20] L. Barack and C. Cutler, Phys. Rev. D 75, 042003 (2007).

[21] O. Dreyer et al., Classical Quantum Gravity 21, 787 (2004).

[22] E. Berti, V. Cardoso, and C. M. Will, Phys. Rev. D 73, 064030 (2006).

[23] E. Berti, J. Cardoso, V. Cardoso, and M. Cavaglia, Phys. Rev. D 76, 104044 (2007).

[24] B.S. Sathyaprakash and B.F. Schutz, Living Rev. Relativity 12, 2 (2009).

[25] F.E. Schunck and E.W. Mielke, Classical Quantum Gravity 20, R301 (2003).

[26] D. F. Torres, S. Capozziello, and G. Lambiase, Phys. Rev. D 62, 104012 (2000).

[27] F. S. Guzman, Phys. Rev. D 73, 021501 (2006).

[28] M. Kesden, J. Gair, and M. Kamionkowski, Phys. Rev. D 71, 044015 (2005).

[29] S. Yoshida, Y. Eriguchi, and T. Futamase, Phys. Rev. D 50, 6235 (1994).

[30] J. Balakrishna, R. Bondarescu, G. Daues, F. Siddhartha Guzman, and E. Seidel, Classical Quantum Gravity 23, 2631 (2006)

[31] E. Berti and V. Cardoso, Int. J. Mod. Phys. D 15, 2209 (2006).

[32] P. O. Mazur and E. Mottola, arXiv:gr-qc/0109035.

[33] G. Chapline, E. Hohlfeld, R. B. Laughlin, and D.I. Santiago, Int. J. Mod. Phys. A 18, 3587 (2003).

[34] P. O. Mazur and E. Mottola, Proc. Natl. Acad. Sci. U.S.A. 101, 9545 (2004).

[35] M. Visser and D. L. Wiltshire, Classical Quantum Gravity 21, 1135 (2004).

[36] C. B. M. H. Chirenti and L. Rezzolla, Classical Quantum Gravity 24, 4191 (2007).

[37] P. P. Fiziev, Classical Quantum Gravity 23, 2447 (2006).

[38] W. Israel, Nuovo Cimento B 44, 1 (1966).
[39] T. Regge and J.A. Wheeler, Phys. Rev. 108, 1063 (1957).

[40] L. Lindblom and S. L. Detweiler, Astrophys. J. Suppl. Ser.. 53, 73 (1983).

[41] S. Detweiler and L. Lindblom, Astrophys. J. 292, 12 (1985).

[42] S. Chandrasekhar and V. Ferrari, Proc. R. Soc. A 432, 247 (1991).

[43] V. Cardoso and M. Cavaglia, Phys. Rev. D 74, 024027 (2006).

[44] M. Abramowitz and I. A. Stegun, Handbook of Mathematical Functions with Formulas, Graphs, and Mathematical Tables (Dover, New York, 1972).

[45] F. J. Zerilli, Phys. Rev. D 2, 2141 (1970).

[46] S. Chandrasekhar, The Mathematical Theory of Black Holes (Clarendon, Oxford, 1992).

[47] M. Leins, H. P. Nollert, and M. H. Soffel, Phys. Rev. D 48, 3467 (1993).

[48] M. Visser, Lorentzian Wormholes: From Einstein to Hawking (AIP Publishing, Melville, NY, 1995).

[49] E. Poisson and M. Visser, Phys. Rev. D 52, 7318 (1995).

[50] O. Benhar, E. Berti, and V. Ferrari, Mon. Not. R. Astron. Soc. 310, 797 (1999).

[51] K. D. Kokkotas, Mon. Not. R. Astron. Soc. 268, 1015 (1994).

[52] W. Press, S. Teukolsky, W. Vetterling, and B. Flannery, Numerical Recipes in $\mathrm{C}++$ (Cambridge University Press, Cambridge, England, 2007), 3rd ed..

[53] N. Andersson, Y. Kojima, and K. D. Kokkotas, Astrophys. J. 462, 855 (1996).

[54] K. D. Kokkotas and B. F. Schutz, Gen. Relativ. Gravit. 18, 913 (1986).

[55] N. Andersson, Gen. Relativ. Gravit. 28, 1433 (1996).

[56] E. Berti, Laurea thesis, 1998 (unpublished).

[57] S. Chandrasekhar and V. Ferrari, Proc. R. Soc. A 434, 449 (1991).

[58] S. Chandrasekhar and V. Ferrari, Proc. R. Soc. A 437, 133 (1992).

[59] E. Berti, V. Cardoso, and P. Pani, Phys. Rev. D 79, 101501 (2009).

[60] V. Cardoso, P. Pani, M. Cadoni, and M. Cavaglia, Phys. Rev. D 77, 124044 (2008).

[61] C. B. M. H. Chirenti and L. Rezzolla, Phys. Rev. D 78, 084011 (2008).

[62] K. D. Kokkotas, J. Ruoff, and N. Andersson, Phys. Rev. D 70, 043003 (2004).

[63] T. Damour and S. N. Solodukhin, Phys. Rev. D 76, 024016 (2007).

[64] Z. Andrade and R.H. Price, Phys. Rev. D 60, 104037 (1999).

[65] V. Ferrari and K. D. Kokkotas, Phys. Rev. D 62, 107504 (2000).

[66] E. Poisson and M. Sasaki, Phys. Rev. D 51, 5753 (1995).

[67] Y. Kojima, Prog. Theor. Phys. 77, 297 (1987).

[68] L. Gualtieri, E. Berti, J. A. Pons, G. Miniutti, and V. Ferrari, Phys. Rev. D 64, 104007 (2001).

[69] J. A. Pons, E. Berti, L. Gualtieri, G. Miniutti, and V. Ferrari, Phys. Rev. D 65, 104021 (2002).

[70] E. Berti, J. A. Pons, G. Miniutti, L. Gualtieri, and V. Ferrari, Phys. Rev. D 66, 064013 (2002). 
[71] T. Tanaka, M. Shibata, M. Sasaki, H. Tagoshi, and T. Nakamura, Prog. Theor. Phys. 90, 65 (1993).

[72] E. Poisson, Phys. Rev. D 47, 1497 (1993).

[73] C. Cutler, E. Poisson, G. J. Sussman, and L. S. Finn, Phys. Rev. D 47, 1511 (1993).

[74] C. Cutler, D. Kennefick, and E. Poisson, Phys. Rev. D 50, 3816 (1994).

[75] E. W. Leaver, Proc. R. Soc. A 402, 285 (1985).
[76] E. W. Leaver, Phys. Rev. D 41, 2986 (1990).

[77] W. Gautschi, SIAM Rev. 9, 24 (1967).

[78] H. S. Wall, Analytic Theory of Continued Fractions (Van Nostrand, Princeton, 1948).

[79] H. Pfister, Classical Quantum Gravity 13, 2267 (1996).

[80] V. Cardoso, P. Pani, M. Cadoni, and M. Cavaglia, Classical Quantum Gravity 25, 195010 (2008). 